\title{
A new formulation of Kapila's five-equation model for compressible two-fluid flow, and its numerical treatment
}

\author{
Jasper J. Kreeft ${ }^{\mathrm{a}, *}$, Barry Koren ${ }^{\mathrm{b}, \mathrm{c}}$ \\ ${ }^{a}$ Delft University of Technology, Faculty of Aerospace Engineering, P.O. Box 5058, 2600 GB Delft, The Netherlands \\ ${ }^{\mathrm{b}}$ Centrum Wiskunde E Informatica, P.O. Box 94079, 1090 GB Amsterdam, The Netherlands \\ ${ }^{\mathrm{c}}$ Leiden University, Mathematical Institute, P.O. Box 9512, 2300 RA Leiden, The Netherlands
}

\section{A R T I C L E I N F O}

\section{Article history:}

Received 24 June 2009

Received in revised form 3 March 2010

Accepted 12 April 2010

Available online 24 April 2010

\section{Keywords:}

Compressible two-fluid flow

Two-fluid mixture flow

Exchange terms

Momentum and energy exchange

Interface capturing

Approximate Riemann solver

Shock tube

Shock-bubble-interaction

\begin{abstract}
A B S T R A C T
A new formulation of Kapila's five-equation model for inviscid, non-heat-conducting, compressible two-fluid flow is derived, together with an appropriate numerical method. The new formulation uses flow equations based on conservation laws and exchange laws only. The two fluids exchange momentum and energy, for which exchange terms are derived from physical laws. All equations are written as a single system of equations in integral form. No equation is used to describe the topology of the two-fluid flow. Relations for the Riemann invariants of the governing equations are derived, and used in the construction of an Osher-type approximate Riemann solver. A consistent finite-volume discretization of the exchange terms is proposed. The exchange terms have distinct contributions in the cell interior and at the cell faces. For the exchange-term evaluation at the cell faces, the same Riemann solver as used for the flux evaluation is exploited. Numerical results are presented for two-fluid shock-tube and shock-bubble-interaction problems, the former also for a two-fluid mixture case. All results show good resemblance with reference results.
\end{abstract}

(c) 2010 Elsevier Inc. All rights reserved.

\section{Introduction}

The research interest in modeling and computing compressible, two-fluid flows is longstanding, as is reflected from the 26-year old review article by Stewart and Wendroff [24], which already contains a wealth of literature on the topic.

These days, to model two-fluid flows, seven-equation models are the most complete. Baer and Nunziato's [4] is the best known model in this class. For both fluids, it contains equations for the quantities mass, momentum and energy, already implying six equations. The seventh equation describes the topology of the flow, e.g., the location and shape of the two-fluid interface. A more recent seven-equation model has been proposed by Romenski et al. [19]. Important modeling and numerical work on seven-equation models has been done by Saurel and Abgrall [20].

Besides completeness, a seven-equation model also implies complexity, both physically and numerically. Since its general physics is not always necessary, simpler and more compact models have been proposed and successfully applied. An elegant hierarchy of reduced models exists, with the numbers of equations ranging from six to three only. Examples of the latter are the homogeneous equilibrium model [5] and the barotropic model described in [26]. For a clear and compact overview over existing reduced two-fluid flow models, we refer to [6].

An important class of reduced models is formed by the five-equation models, in which velocity equilibrium and pressure equilibrium are considered, due to zero relaxation time. Both equilibria are valid across two-fluid interfaces modeled as a

\footnotetext{
* Corresponding author. Tel.: +31 152784215; fax: +31 152787077.

E-mail addresses: j.j.kreeft@tudelft.nl (J.J. Kreeft), barry.koren@cwi.nl (B. Koren).
} 
contact discontinuity, a practically relevant case. The archetype five-equation model is that of Kapila et al. [10]. It has already found many applications, a non-exhaustive list of excellent references is [12,3,14,2,21,17]. (Recently, Saurel et al. have even derived a six-equation model from a five-equation one [22].) Kapila's five-equation model contains four equations for conservative quantities: two for mass (e.g., bulk mass and mass of one of the two fluids), one for bulk momentum, and one for bulk energy. Like the Baer-Nunziato model, it is also completed by an equation for a non-conservative quantity describing the flow topology, in the Kapila model, a non-homogeneous convection equation for the volume fraction of one of the two fluids.

In the present paper, we derive a new formulation of Kapila's five-equation model. The first four equations are the same as in Kapila's five-equation model, our fifth equation, which substitutes the topological equation from Kapila's formulation, is new. It is the energy equation for one of the two fluids. In [22], the energy equation for one of the two fluids is also considered, though in a six-equation context. The advantage of the current new five-equation formulation is that all equations can now be written in integral form, in a single system. Our fifth equation contains a righthand side that describes the exchange of energy between the two fluids, in terms of rate of work. Whereas the first four equations are conservation laws, the fifth equation is an exchange law; it is conservation-law-like though. As a consequence, the entire system allows for a rather straightforward derivation and application of finite-volume methods and corresponding numerical tools, such as approximate Riemann solvers. In the current manuscript, an Osher-type approximate Riemann solver is derived for the new fiveequation formulation. For the numerical treatment of the righthand side in the fifth equation, the energy-exchange terms, use is also made of the just derived approximate Riemann solver.

In differential form, the present formulation is actually identical to Kapila's. However, in the form in which it is derived, discretized and solved, the predominant integral form and corresponding finite-volume method, it is different; its fifth equation is new and consistent with the other four equations.

The contents of the paper is the following. In Section 2, the new five-equation formulation is derived. Most attention is for the derivation and physical interpretation of the energy-exchange terms. In Section 3, the numerical method for the fiveequation model is presented, with - also here - most attention for the energy-exchange terms. In Section 4, numerical results are presented, for three shock-tube problems and two standard shock-bubble-interaction problems. Section 5 concludes the paper.

\section{New five-equation formulation}

\subsection{Assumptions}

Each of the two fluids is assumed to be mass conservative. They may exchange momentum by exerting forces on each other, and may exchange energy due to work. The amount of momentum and energy exchange depends on the relaxation speed of pressure and velocity. An important assumption is instantaneous relaxation of the pressure and the velocity vector, i.e.,

$$
\begin{aligned}
& p_{1}=p_{2} \equiv p, \\
& \boldsymbol{u}_{1}=\boldsymbol{u}_{2} \equiv \boldsymbol{u},
\end{aligned}
$$

where the subscripts refer to the two fluids. Assumption (1) prescribes that the pressures and velocity vectors on both sides of a two-fluid interface are equal. It is the known step to reduce a seven-equation model to a five-equation model, see, e.g., $[1,20,10,3,14,6]$. Further, viscosity and heat conduction are neglected. Since this implies that the two fluids do not exchange heat, in general, there will be no thermal equilibrium.

\subsection{Conservation and exchange laws}

Consider a control volume $V$, which is fixed in space and time. For this volume three physical principles are known: conservation of bulk mass, bulk momentum and bulk energy, in integral form written as:

$$
\begin{aligned}
& \frac{\partial}{\partial t} \int_{V} \rho \mathrm{d} V+\oint_{S} \rho \boldsymbol{u} \cdot \boldsymbol{n} \mathrm{d} S=0, \\
& \frac{\partial}{\partial t} \int_{V} \rho \boldsymbol{u} \mathrm{d} V+\oint_{S} \rho \boldsymbol{u} \otimes \boldsymbol{u} \cdot \boldsymbol{n} \mathrm{d} S+\oint_{S} p \boldsymbol{n} \mathrm{d} S=\mathbf{0}, \\
& \frac{\partial}{\partial t} \int_{V} \rho E \mathrm{~d} V+\oint_{S} \rho E \boldsymbol{u} \cdot \boldsymbol{n} \mathrm{d} S+\oint_{S} p \boldsymbol{u} \cdot \boldsymbol{n} \mathrm{d} S=0 .
\end{aligned}
$$

There can be two fluids in the control volume $V$. The pressure and velocity are equal over a two-fluid interface, but the density and total energy do not need to be so; in general $\rho_{1} \neq \rho_{2}$ and $E_{1} \neq E_{2}$. The quantities $\rho$ and $E$ in Eqs. (2) and (3) are the bulk density and bulk total energy.

For each fluid separately an equation for mass, momentum and energy can also be written. The mass of each fluid is conserved. The conservation of, e.g., mass of fluid 1 can be written as 


$$
\frac{\partial}{\partial t} \int_{V_{1}(t)} \rho_{1} \mathrm{~d} V_{1}+\oint_{S_{1}(t)} \rho_{1}\left(\boldsymbol{u}-\boldsymbol{u}_{S_{1}}\right) \cdot \boldsymbol{n} \mathrm{d} S_{1}=0
$$

where the volume $V_{1}(t)$ and the surface $S_{1}(t)$ are unknown and may vary in time due to a possible motion of the interface with velocity $\boldsymbol{u}_{S_{1}}$. The momentum equation of fluid 1 is not conservative; fluid 2 can exert a force on fluid 1 and vice versa. We denote the net force per unit of volume exerted on fluid 1 by $\mathbf{F}$. Since $\mathbf{F}$ is a result of both fluids, it is integrated over the total volume $V$. Hence, the momentum equation of fluid 1 can be written as

$$
\frac{\partial}{\partial t} \int_{V_{1}(t)} \rho_{1} \boldsymbol{u} \mathrm{d} V_{1}+\oint_{S_{1}(t)} \rho_{1} \boldsymbol{u} \otimes\left(\boldsymbol{u}-\boldsymbol{u}_{S_{1}}\right) \cdot \boldsymbol{n} \mathrm{d} S_{1}+\oint_{S_{1}(t)} p \boldsymbol{n} \mathrm{d} S_{1}=\int_{V} \mathbf{F} \mathrm{d} V .
$$

An expression for $\mathbf{F}$ in terms of the existing unknowns will be derived in Section 2.3. There it will also be shown that the resulting momentum equation of fluid 1 is a dependent equation; it can be directly derived from bulk mass Eq. (2), bulk momentum Eq. (3) and mass Eq. (5). The cause of this dependence is the assumption that pressure and velocity are equal over two-fluid interfaces. This is in contrast with the energy equation of fluid 1 , which will be independent, because we do not assume temperature equilibrium.

The force $\mathbf{F}$ also contributes to the energy equation of fluid 1 in the form of rate of mechanical work performed per unit of volume:

$$
\dot{W}_{\mathrm{M}}=\mathbf{F} \cdot \boldsymbol{u}
$$

Besides mechanical work, also thermodynamic work may be performed. The rate of thermodynamic work per unit of volume is denoted by $\dot{W}_{\mathrm{T}}$. The energy equation of fluid 1 then becomes:

$$
\frac{\partial}{\partial t} \int_{V_{1}(t)} \rho_{1} E_{1} \mathrm{~d} V_{1}+\oint_{S_{1}(t)} \rho_{1} E_{1}\left(\boldsymbol{u}-\boldsymbol{u}_{S_{1}}\right) \cdot \boldsymbol{n} \mathrm{d} S_{1}+\oint_{S_{1}(t)} p \boldsymbol{u} \cdot \boldsymbol{n} \mathrm{d} S_{1}=\int_{V}\left(\dot{W}_{\mathrm{M}}+\dot{W}_{\mathrm{T}}\right) \mathrm{d} V
$$

Besides for $\mathbf{F}$, and hence $\dot{W}_{\mathrm{M}}$, in Section 2.3, also for $\dot{W}_{\mathrm{T}}$ an expression in terms of existing variables will be derived. The exchange term $\int_{V}\left(\dot{W}_{\mathrm{M}}+\dot{W}_{\mathrm{T}}\right) \mathrm{d} V$ is the net rate of work performed by fluid 2 on fluid 1 . By applying the divergence theorem to the surface integrals of Eqs. (5), (6) and (8), the unknown integration parameter $S_{1}(t)$ disappears, still leaving $V_{1}(t)$ as an unknown.

Several methods exist to find the interface location and thus $V_{1}(t)$. An important class is formed by the level-set methods - see the archival paper [13] and the monographs [15,23] - which are interface-tracking methods. Interface tracking requires an extra transport equation. Here we choose the interface-capturing approach. For this purpose, the volume fraction of fluid 1 ,

$$
\alpha=\frac{V_{1}}{V}
$$

is introduced, to enable integration over the volume $V$ instead of over $V_{1}(t)$, simplifying Eqs. (5) and (8) to:

$$
\begin{aligned}
& \frac{\partial}{\partial t} \int_{V} \alpha \rho_{1} \mathrm{~d} V+\int_{V} \nabla \cdot \alpha \rho_{1} \boldsymbol{u} \mathrm{d} V=0, \\
& \frac{\partial}{\partial t} \int_{V} \alpha \rho_{1} E_{1} \mathrm{~d} V+\int_{V} \nabla \cdot \alpha \rho_{1} E_{1} \boldsymbol{u} \mathrm{d} V+\int_{V} \nabla \cdot \alpha p \boldsymbol{u} \mathrm{d} V=\int_{V}\left(\dot{W}_{\mathrm{M}}+\dot{W}_{\mathrm{T}}\right) \mathrm{d} V .
\end{aligned}
$$

The same kind of equations can be derived for fluid 2 , with $1-\alpha$ the volume fraction of fluid 2 . By adding these equations for fluid 2 to Eqs. (10) and (11), they must result in the bulk Eqs. (2) and (4), implying the following expressions for bulk density and bulk total energy:

$$
\begin{aligned}
& \rho=\alpha \rho_{1}+(1-\alpha) \rho_{2}, \\
& \rho E=\alpha \rho_{1} E_{1}+(1-\alpha) \rho_{2} E_{2},
\end{aligned}
$$

with the total energy for each fluid defined as

$$
\begin{aligned}
& E_{1}=e_{1}+\frac{1}{2} \boldsymbol{u} \cdot \boldsymbol{u} \\
& E_{2}=e_{2}+\frac{1}{2} \boldsymbol{u} \cdot \boldsymbol{u} .
\end{aligned}
$$

Through the equations of state, the internal energies $e_{1}$ and $e_{2}$ can be written as functions of the density of the corresponding fluid and the pressure:

$$
\begin{aligned}
& e_{1}=e_{1}\left(\rho_{1}, p\right), \\
& e_{2}=e_{2}\left(\rho_{2}, p\right) .
\end{aligned}
$$

The bulk internal energy $e$ is defined by

$$
\rho e=\alpha \rho_{1} e_{1}+(1-\alpha) \rho_{2} e_{2} .
$$


This consistently implies

$$
E=e+\frac{1}{2} \boldsymbol{u} \cdot \boldsymbol{u}
$$

As soon as the energy-exchange terms $\dot{W}_{\mathrm{M}}$ and $\dot{W}_{\mathrm{T}}$ are expressed in terms of already introduced variables (in primitive form: $p, \boldsymbol{u}, \rho_{1}, \rho_{2}$ and $\alpha$ ), the system of equations is closed. In differential form, the practical form for deriving the rate-ofwork terms $\dot{W}_{\mathrm{M}}$ and $\dot{W}_{\mathrm{T}}$, the system reads:

$$
\begin{aligned}
& \frac{\partial \rho}{\partial t}+\nabla \cdot(\rho \boldsymbol{u})=0, \\
& \frac{\partial(\rho \boldsymbol{u})}{\partial t}+\nabla \cdot(\rho \boldsymbol{u} \otimes \boldsymbol{u})+\nabla p=\mathbf{0}, \\
& \frac{\partial(\rho E)}{\partial t}+\nabla \cdot(\rho E \boldsymbol{u})+\nabla \cdot(p \boldsymbol{u})=0, \\
& \frac{\partial\left(\alpha \rho_{1}\right)}{\partial t}+\nabla \cdot\left(\alpha \rho_{1} \boldsymbol{u}\right)=0, \\
& \frac{\partial\left(\alpha \rho_{1} E_{1}\right)}{\partial t}+\nabla \cdot\left(\alpha \rho_{1} E_{1} \boldsymbol{u}\right)+\nabla \cdot(\alpha p \boldsymbol{u})=\dot{W}_{\mathrm{M}}+\dot{W}_{\mathrm{T}} .
\end{aligned}
$$

These equations may be cast into the compact form

$$
\frac{\partial \mathbf{q}}{\partial t}+\nabla \cdot \mathcal{F}(\mathbf{q})=\mathbf{S}(\mathbf{q})
$$

\subsection{Rate-of-work terms}

The derivation of the energy-exchange terms is done for flow solutions for which first-order spatial derivatives are assumed to exist.

\subsubsection{Mechanical work}

The rate of mechanical work $\dot{W}_{\mathrm{M}}$ is derived using velocity-equilibrium relation (1b). We start by considering the momentum equation of fluid 1 in differential form:

$$
\frac{\partial\left(\alpha \rho_{1} \boldsymbol{u}\right)}{\partial t}+\nabla \cdot\left(\alpha \rho_{1} \boldsymbol{u} \otimes \boldsymbol{u}\right)+\nabla(\alpha p)=\mathbf{F} .
$$

Here it already appears that momentum Eq. (20) is a dependent equation, because it does not give new time-derivative information; $\frac{\partial\left(\alpha \rho_{1}\right)}{\partial t}$ is already described by Eq. (18d), and $\frac{\partial u}{\partial t}$ by (18a) and (18b). The purpose of Eq. (20) is to derive an expression for the force $\mathbf{F}$.

By expanding (20) and substituting (18d), we get the primitive equation

$$
\frac{\mathrm{D} \boldsymbol{u}}{\mathrm{D} t}+\frac{1}{\alpha \rho_{1}} \nabla(\alpha p)=\frac{1}{\alpha \rho_{1}} \mathbf{F} .
$$

The similar can be done for bulk momentum Eq. (18b); expanding (18b) and substituting (18a), we find:

$$
\frac{\mathrm{D} \boldsymbol{u}}{\mathrm{D} t}+\frac{1}{\rho} \nabla p=0
$$

Eliminating $\frac{\mathrm{D} u}{\mathrm{D} t}$ from (21) and (22), we get

$$
\mathbf{F}=\nabla(\alpha p)-\frac{\alpha \rho_{1}}{\rho} \nabla p=p \nabla \alpha+(\alpha-\beta) \nabla p
$$

where an additional symbol has been introduced, $\beta$, the mass fraction of fluid 1 ,

$$
\beta=\frac{\alpha \rho_{1}}{\rho} .
$$

Hence, the rate of energy exchange due to mechanical work can be expressed in terms of the existing variables as

$$
\dot{W}_{\mathrm{M}}=p \boldsymbol{u} \cdot \nabla \alpha+(\alpha-\beta) \boldsymbol{u} \cdot \nabla p .
$$

The two force terms in the righthand side of (23) can still be interpreted.

The term $p \nabla \alpha$ is best explained by making a comparison with quasi-1D channel flow, Fig. 1(a). For a channel flow with variable area, it can be written 


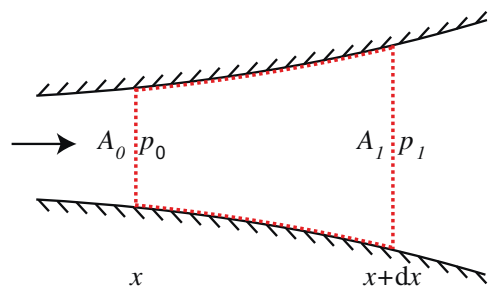

(a)

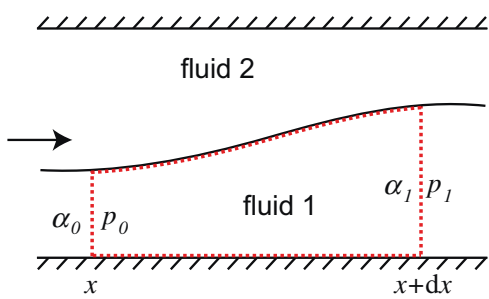

(b)

Fig. 1. Similarity between quasi-1D channel flow (a) and the two-fluid flow model (b).

$$
\oint_{S} p \boldsymbol{n} \mathrm{d} S=p_{1} A_{1}-p_{0} A_{0}-\int_{0}^{1} p \mathrm{~d} A,
$$

with $S$ the closed, red contour. In differential form this becomes

$$
p \boldsymbol{n} \mathrm{d} S=\mathrm{d}(p A)-p \mathrm{~d} A .
$$

Analogously, in our two-fluid model, Fig. 1(b), we have the forces $\nabla(\alpha p)$ and $p \nabla \alpha$. The former represents the net pressure force at the two vertical faces, at $x$ and $x+\mathrm{d} x$. The latter is the pressure force acting on the upper (in this example curvilinear) two-fluid interface in between $x$ and $x+\mathrm{d} x$.

To interpret the term $(\alpha-\beta) \nabla p$, consider for example an expansion. Then we know that the pressure drops (dp $<0)$ and the velocity rises $(\mathrm{d} \boldsymbol{u}>0)$. Newton's second law of motion tells that the amount of acceleration of each of the two fluids is inversely proportional to their densities. So when the density of fluid 1 is for instance lower than that of fluid $2, \beta<\alpha$, then the velocity of fluid 1 tends to become higher than that of fluid 2 . The force $(\alpha-\beta) \nabla p$, exerted by fluid 2 on fluid 1 , prevents this; it is the force that ensures velocity equilibrium.

\subsubsection{Thermodynamic work}

For the derivation of the rate of thermodynamic work $\dot{W}_{\mathrm{T}}$, and also for further analysis purposes, first some other primitive equations are derived.

2.3.2.1. More primitive equations. With Eqs. (17), (18a) and (22), Eq. (18c) for the bulk energy is rewritten as

$$
\frac{\mathrm{D} e}{\mathrm{D} t}+\frac{p}{\rho} \nabla \cdot \boldsymbol{u}=0 .
$$

For the densities $\rho_{1}$ and $\rho_{2}$, the following two primitive equations hold:

$$
\begin{aligned}
& \frac{\mathrm{D} \rho_{1}}{\mathrm{D} t}+\frac{\rho_{1}}{\alpha} \frac{\mathrm{D} \alpha}{\mathrm{D} t}+\rho_{1} \nabla \cdot \boldsymbol{u}=0, \\
& \frac{\mathrm{D} \rho_{2}}{\mathrm{D} t}-\frac{\rho_{2}}{1-\alpha} \frac{\mathrm{D} \alpha}{\mathrm{D} t}+\rho_{2} \nabla \cdot \boldsymbol{u}=0 .
\end{aligned}
$$

And Eqs. (18a), (18d) and (24) result in the primitive equation for the mass fraction:

$$
\frac{\mathrm{D} \beta}{\mathrm{D} t}=0 .
$$

Hence, the mass fraction is convected with the flow. This is obvious, a fluid blob (possibly containing both fluids) remains to contain the same fluid particles over time. Finally, with Eqs. (14a), (18d), (22), (24) and (25), from (18e) the following primitive equation is derived for the internal energy $e_{1}$ :

$$
\frac{\mathrm{D} e_{1}}{\mathrm{D} t}+\frac{p}{\rho_{1}} \nabla \cdot \boldsymbol{u}=\frac{\dot{W}_{\mathrm{T}}}{\alpha \rho_{1}} .
$$

Similarly, for fluid 2 we find:

$$
\frac{\mathrm{D} e_{2}}{\mathrm{D} t}+\frac{p}{\rho_{2}} \nabla \cdot \boldsymbol{u}=-\frac{\dot{W}_{\mathrm{T}}}{(1-\alpha) \rho_{2}} .
$$

2.3.2.2. Isentropic compressibility relations. Here, non-adiabatic processes, like external heating, are not considered, as are viscosity, heat conduction and discontinuities. Hence, the two fluids flow isentropicly; 


$$
\begin{aligned}
& \frac{\mathrm{D} s_{1}}{\mathrm{D} t}=0, \\
& \frac{\mathrm{D} s_{2}}{\mathrm{D} t}=0 .
\end{aligned}
$$

In [14] these relations follow from a reduction of the seven-equation Baer-Nunziato model.

Eq. (32) allows us to also derive primitive equations for the pressure and volume fraction. The pressure can be expressed in two independent thermodynamic variables. At first the pressure is expressed as a function of the entropy and the density of both fluids: $p=p\left(s_{1}, \rho_{1}\right), p=p\left(s_{2}, \rho_{2}\right)$. Taking the total derivative of the pressure and substituting Eq. (32), we get

$$
\begin{aligned}
& \frac{\mathrm{D} p}{\mathrm{D} t}=\left(\frac{\partial p}{\partial \rho_{1}}\right)_{s_{1}} \frac{\mathrm{D} \rho_{1}}{\mathrm{D} t}=c_{1}^{2} \frac{\mathrm{D} \rho_{1}}{\mathrm{D} t}, \\
& \frac{\mathrm{D} p}{\mathrm{D} t}=\left(\frac{\partial p}{\partial \rho_{2}}\right)_{s_{2}} \frac{\mathrm{D} \rho_{2}}{\mathrm{D} t}=c_{2}^{2} \frac{\mathrm{D} \rho_{2}}{\mathrm{D} t},
\end{aligned}
$$

with $c_{1}$ and $c_{2}$ the speeds of sound of both fluids. With (29a) and (29b), respectively, (33a) and (33b) can be rewritten as:

$$
\begin{aligned}
& \frac{\mathrm{D} p}{\mathrm{D} t}+\rho_{1} c_{1}^{2}\left(\frac{1}{\alpha} \frac{\mathrm{D} \alpha}{\mathrm{D} t}+\nabla \cdot \boldsymbol{u}\right)=0, \\
& \frac{\mathrm{D} p}{\mathrm{D} t}+\rho_{2} c_{2}^{2}\left(\frac{-1}{1-\alpha} \frac{\mathrm{D} \alpha}{\mathrm{D} t}+\nabla \cdot \boldsymbol{u}\right)=0 .
\end{aligned}
$$

By eliminating the pressure derivatives, the primitive equation for the volume fraction is found:

$$
\frac{\mathrm{D} \alpha}{\mathrm{D} t}+\varphi \nabla \cdot \boldsymbol{u}=0, \quad \varphi=\alpha(1-\alpha) \frac{\frac{1}{\rho_{2} c_{2}^{2}}-\frac{1}{\rho_{1} c_{1}^{2}}}{\frac{\alpha}{\rho_{1} c_{1}^{2}}+\frac{1-\alpha}{\rho_{2} c_{2}^{2}}} .
$$

This is the fifth equation in Kapila's model. It shows that in differential form, the current model is equal to Kapila's model.

Let us take a closer look at Eq. (35). We follow a fluid blob containing both fluids. To what extent the volume of each separate fluid in the blob will change under compression or expansion depends on the compressibilities of the fluids. The terms $\frac{1}{\rho_{1} c_{1}^{2}}$ and $\frac{1}{\rho_{2} c_{2}^{2}}$ are the isentropic compressibilities, $\tau_{1}$ and $\tau_{2}$, of both fluids:

$$
\begin{aligned}
& \tau_{1}=\frac{1}{\rho_{1}}\left(\frac{\partial \rho_{1}}{\partial p}\right)_{s_{1}}=\frac{1}{\rho_{1} c_{1}^{2}}, \\
& \tau_{2}=\frac{1}{\rho_{2}}\left(\frac{\partial \rho_{2}}{\partial p}\right)_{s_{2}}=\frac{1}{\rho_{2} c_{2}^{2}} .
\end{aligned}
$$

Defining the bulk isentropic compressibility as

$$
\tau=\alpha \tau_{1}+(1-\alpha) \tau_{2}
$$

(35) can be compactly written as

$$
\frac{\mathrm{D} \alpha}{\mathrm{D} t}+\alpha(1-\alpha) \frac{\tau_{2}-\tau_{1}}{\tau} \nabla \cdot \boldsymbol{u}=0
$$

Eq. (38) shows that a difference in compressibility of the two fluids $\left(\tau_{2}-\tau_{1} \neq 0\right)$, under compression or expansion $(\nabla \cdot \boldsymbol{u} \neq 0)$, causes a change in volume fraction. As an example, suppose the flow element expands, then $\nabla \cdot \boldsymbol{u}>0$. If fluid 1 is more compressible than fluid 2 , so $\tau_{1}>\tau_{2}$, then the volume fraction of fluid 1 must increase, $\frac{\mathrm{D} \alpha}{\mathrm{D} t}>0$. Pressure equilibrium under compression or expansion is ensured by a change in volume fraction.

Finally, as the primitive equation for the pressure it follows by elimination of $\frac{\mathrm{D} \alpha}{\mathrm{Dt}}$ from (34):

$$
\frac{\mathrm{D} p}{\mathrm{D} t}+\frac{1}{\tau} \nabla \cdot \boldsymbol{u}=\frac{\mathrm{D} p}{\mathrm{D} t}+\rho c^{2} \nabla \cdot \boldsymbol{u}=0
$$

where we have written the bulk compressibility $\tau$ in terms of the bulk density $\rho$ and a bulk speed of sound $c$. With (36) and (37) it follows that $c$ is implicitly defined through the harmonic mean

$$
\frac{1}{\rho c^{2}}=\frac{\alpha}{\rho_{1} c_{1}^{2}}+\frac{1-\alpha}{\rho_{2} c_{2}^{2}}
$$

This relation was also found and described in $[10,14]$.

2.3.2.3. Thermodynamic work relations. We now choose to express the pressure in terms of the density and internal energy of both fluids: $p=p\left(e_{1}, \rho_{1}\right), p=p\left(e_{2}, \rho_{2}\right)$. It holds:

$$
\frac{\mathrm{D} p}{\mathrm{D} t}\left(e_{1}, \rho_{1}\right)=\frac{\mathrm{D} p}{\mathrm{D} t}\left(e_{2}, \rho_{2}\right) \text {. }
$$


The pressure derivatives can be expanded into the thermodynamic variables:

$$
\left(\frac{\partial p}{\partial e_{1}}\right)_{\rho_{1}} \frac{\mathrm{D} e_{1}}{\mathrm{D} t}+\left(\frac{\partial p}{\partial \rho_{1}}\right)_{e_{1}} \frac{\mathrm{D} \rho_{1}}{\mathrm{D} t}=\left(\frac{\partial p}{\partial e_{2}}\right)_{\rho_{2}} \frac{\mathrm{D} e_{2}}{\mathrm{D} t}+\left(\frac{\partial p}{\partial \rho_{2}}\right)_{e_{2}} \frac{\mathrm{D} \rho_{2}}{\mathrm{D} t} .
$$

With Eqs. (29) and (31), this can be rewritten as

$$
\begin{aligned}
& \dot{W}_{\mathrm{T}}\left(\frac{1}{\alpha \rho_{1}}\left(\frac{\partial p}{\partial e_{1}}\right)_{\rho_{1}}+\frac{1}{(1-\alpha) \rho_{2}}\left(\frac{\partial p}{\partial e_{2}}\right)_{\rho_{2}}\right)=\frac{\mathrm{D} \alpha}{\mathrm{D} t}\left(\frac{\rho_{1}}{\alpha}\left(\frac{\partial p}{\partial \rho_{1}}\right)_{e_{1}}+\frac{\rho_{2}}{1-\alpha}\left(\frac{\partial p}{\partial \rho_{2}}\right)_{e_{2}}\right) \\
& +\left(\rho_{1}\left(\frac{\partial p}{\partial \rho_{1}}\right)_{e_{1}}+\frac{p}{\rho_{1}}\left(\frac{\partial p}{\partial e_{1}}\right)_{\rho_{1}}-\rho_{2}\left(\frac{\partial p}{\partial \rho_{2}}\right)_{e_{2}}-\frac{p}{\rho_{2}}\left(\frac{\partial p}{\partial e_{2}}\right)_{\rho_{2}}\right) \nabla \cdot \boldsymbol{u} .
\end{aligned}
$$

Again we make use of the speed of sound of each fluid, but now with the current thermodynamic variables used in the equations of state,

$$
\begin{aligned}
& c_{1}^{2}=\left(\frac{\partial p}{\partial \rho_{1}}\right)_{s_{1}}=\left(\frac{\partial p}{\partial \rho_{1}}\right)_{e_{1}}+\frac{p}{\rho_{1}^{2}}\left(\frac{\partial p}{\partial e_{1}}\right)_{\rho_{1}}, \\
& c_{2}^{2}=\left(\frac{\partial p}{\partial \rho_{2}}\right)_{s_{2}}=\left(\frac{\partial p}{\partial \rho_{2}}\right)_{e_{2}}+\frac{p}{\rho_{2}^{2}}\left(\frac{\partial p}{\partial e_{2}}\right)_{\rho_{2}} .
\end{aligned}
$$

From (43), with (44) and primitive Eq. (35) for $\alpha$, it then formally follows:

$$
\dot{W}_{\mathrm{T}}=-p \frac{\mathrm{D} \alpha}{\mathrm{D} t}
$$

The term $-p \frac{\mathrm{D} \alpha}{\mathrm{Dt}}$ clearly is a rate of thermodynamic work term. It is the rate of work performed on the two-fluid interface, which causes the interface to move such that pressure equilibrium under compression or expansion is ensured. With Eq. (38), expression (45) for $\dot{W}_{\mathrm{T}}$ can be rewritten in terms of spatial derivatives only:

$$
\dot{W}_{\mathrm{T}}=p \alpha(1-\alpha) \frac{\tau_{2}-\tau_{1}}{\tau} \nabla \cdot \boldsymbol{u}
$$

\subsubsection{Complete rate-of-work term and remarks about discontinuities}

Addition of Eqs. (25) and (46) finally gives us the total rate of energy exchange per unit volume between fluid 2 and fluid 1, expressed in existing variables:

$$
\dot{W}=\dot{W}_{\mathrm{M}}+\dot{W}_{\mathrm{T}}=p \boldsymbol{u} \cdot \nabla \alpha+(\alpha-\beta) \boldsymbol{u} \cdot \nabla p+p \alpha(1-\alpha) \frac{\tau_{2}-\tau_{1}}{\tau} \nabla \cdot \boldsymbol{u}
$$

The model derived so far is not valid across discontinuities, where locally the derivatives of the state variables do not exist. To allow discontinuities in the flow, jump conditions have to be derived that describe the behavior of the two-fluid flow over a discontinuity. This is not as straightforward as it is in single-fluid Euler flow. For the present five-equation model, jump relations are derived in chapter 6 of [28].

Our numerical treatment of the rate-of-work terms, to be presented in the next section, does not need jump conditions for shock waves though. It makes a neat, smooth approximation of shock waves (overturned compression waves) by using an Osher-type Riemann solver in solution space.

\section{Numerical method}

\subsection{Equations}

We consider the entire system of equations in integral form, i.e., (19) is rewritten as:

$$
\frac{\partial}{\partial t} \int_{\Omega} \boldsymbol{q} \mathrm{d} \Omega+\oint_{\Gamma} \mathcal{F} \cdot \boldsymbol{n} \mathrm{d} \Gamma=\int_{\Omega} \mathbf{S} \mathrm{d} \Omega
$$

with, in $3 \mathrm{D}$, writing the velocity vector in its three components, $\boldsymbol{u}=(u, v, w)$ :

$$
\boldsymbol{q}=\left(\begin{array}{c}
\rho \\
\rho u \\
\rho v \\
\rho w \\
\rho E \\
\alpha \rho_{1} \\
\alpha \rho_{1} E_{1}
\end{array}\right),
$$




$$
\begin{aligned}
& \mathcal{F}(\boldsymbol{q})=(\mathbf{f}(\boldsymbol{q}), \mathbf{g}(\boldsymbol{q}), \mathbf{h}(\boldsymbol{q})), \\
& \begin{array}{c}
\mathbf{f}=\left(\begin{array}{c}
\rho u \\
\rho u^{2}+p \\
\rho u v \\
\rho u w \\
\rho u\left(E+\frac{p}{\rho}\right) \\
\alpha \rho_{1} u \\
\alpha \rho_{1} u\left(E_{1}+\frac{p}{\rho_{1}}\right)
\end{array}\right), \quad \mathbf{g}=\left(\begin{array}{c}
\rho v \\
\rho v u \\
\rho v^{2}+p \\
\rho v w \\
\rho v\left(E+\frac{p}{\rho}\right) \\
\alpha \rho_{1} v \\
\alpha \rho_{1} v\left(E_{1}+\frac{p}{\rho_{1}}\right) \\
0 \\
0 \\
0 \\
0 \\
0 \\
0 \\
0 \\
\dot{W}
\end{array}\right)=\left(\begin{array}{c}
\rho w \\
\rho w u \\
\rho w v \\
\rho w^{2}+p \\
\rho w\left(E+\frac{p}{\rho}\right) \\
\alpha \rho_{1} w \\
\alpha \rho_{1} w\left(E_{1}+\frac{p}{\rho_{1}}\right) \\
0 \\
0 \\
0 \\
p \boldsymbol{h} \cdot \nabla \alpha+(\alpha-\beta) \boldsymbol{u} \cdot \nabla p+p \alpha(1-\alpha) \frac{\tau_{2}-\tau_{1}}{\tau} \nabla \cdot \boldsymbol{u}
\end{array}\right),
\end{array}
\end{aligned}
$$

In our numerical method, $\Omega$ is a cell-centered finite volume, fixed in space, with contour $\Gamma$ and unit normal $\boldsymbol{n}=\left(n_{x}, n_{y}, n_{z}\right)$ on $\Gamma$. We proceed by describing the numerical treatment of the lefthand side of this equation, in Section 3.2, and next the righthand side, in Section 3.3.

\subsection{Cell-face state construction and time integration}

The spatial and temporal discretization is treated with well-proven numerical methods. For the evaluation of the states at the cell faces an Osher-type approximate Riemann solver [16] is constructed, in the so-called P(hysical) variant [8]. The Riemann solver gets limited higher-order accurate left and right cell-face states as input (MUSCL approach). The limiter applied is the one of the second author [11]. It corresponds to the maximal possible extent with Van Leer's $\kappa=\frac{1}{3}$ scheme [27], within Sweby's TVD domain [25]. Its accuracy properties are known to be good [29]. Formally, in 1D, this MUSCL approach is thirdorder accurate [11].

\subsubsection{Cell-face state construction using Osher's Riemann solver}

Consider the set of transport Eqs. (18a), (22), (30), (35) and (39), and write it for the 1D case in the form

$$
\frac{\partial \boldsymbol{w}}{\partial t}+A(\boldsymbol{w}) \frac{\partial \boldsymbol{w}}{\partial x}=0
$$

with

$$
\boldsymbol{w}=\left(\begin{array}{c}
\rho \\
u \\
p \\
\beta \\
\alpha
\end{array}\right), \quad A(\boldsymbol{w})=\left(\begin{array}{ccccc}
u & \rho & 0 & 0 & 0 \\
0 & u & \frac{1}{\rho} & 0 & 0 \\
0 & \rho c^{2} & u & 0 & 0 \\
0 & 0 & 0 & u & 0 \\
0 & \varphi & 0 & 0 & u
\end{array}\right)
$$

Hence, the full (1D) system is hyperbolic with wave speeds

$$
\lambda_{1}=u-c, \quad \lambda_{2,3,4}=u, \quad \lambda_{5}=u+c .
$$

A finite volume containing both fluids, has a single speed of sound, the bulk speed of sound. In the seven-equation model of Baer and Nunziato [4], two different speeds of sound may occur in a single finite volume. The wave speeds (51) are the same as in Kapila's model [10], of course, and the same as in the reduced model of [14].

The Riemann invariants $\psi_{k}$ of the system of equations are prescribed by the following set of differential equations:

$$
\mathrm{d} \psi_{1}=\mathrm{d} u-\frac{\mathrm{d} p}{\rho c}, \quad \mathrm{~d} \psi_{2}=\mathrm{d} p-c^{2} \mathrm{~d} \rho, \quad \mathrm{d} \psi_{3}=\mathrm{d} \beta, \mathrm{d} \psi_{4}=\rho \mathrm{d} \alpha-\varphi \mathrm{d} \rho, \quad \mathrm{d} \psi_{5}=\mathrm{d} u+\frac{\mathrm{d} p}{\rho c} .
$$

Three of the five equations, those for $\psi_{1}, \psi_{2}$ and $\psi_{5}$, also occur in the single-fluid Euler model. The equations for $\psi_{3}$ and $\psi_{4}$ contain the fraction variables that are specific for this two-fluid model. The Riemann invariants $\psi_{3}$ and $\psi_{4}$ show again that the mass fraction is convected with the flow, whereas the volume fraction is not.

Along the wave path in solution space, with the subpaths in P-variant ordering [8], the Riemann invariants are distributed as shown in Fig. 2. 
Unlike the Riemann invariants of, e.g., the single-fluid, perfect-gas Euler equations, it is not possible to write explicit expressions for the current Riemann invariants $\psi_{k}$, along all three subpaths of the wavepath, not even for perfect gases. For the middle subpath we can, but for the left and right subpath we can not. One could argue: why not use d $\psi_{2}=\mathrm{d} s$ and $\mathrm{d} \psi_{4}=\mathrm{d} s_{1}$ instead, with $s=\beta s_{1}+(1-\beta) s_{2}$, as is done in [14]. Since $c$ is a function of $\rho$ and $\alpha$ among others, for perfect gases already, expressions along $\lambda_{1,5}$ for $\rho$ and $\alpha$ would have to be extracted then from $s_{i}=$ constant with $s_{i} \sim \ln \left(p / \rho_{i}^{\gamma(\alpha)}\right)$ for $i=1$, 2 , a coupled system of implicit algebraic nonlinear equations.

We rewrite (52) into a system of ordinary differential equations which has to be solved numerically to compute the intermediate solutions $\boldsymbol{q}^{\mathrm{LM}}$ and $\boldsymbol{q}^{\mathrm{RM}}$ (Fig. 3). As independent variable for the system of differential equations, we may choose the velocity component $u$. This results in:

$$
\frac{\mathrm{d} p}{\mathrm{~d} u}= \pm \rho c, \quad \frac{\mathrm{d} \rho}{\mathrm{d} u}= \pm \frac{\rho}{c}, \quad \frac{\mathrm{d} \alpha}{\mathrm{d} u}= \pm \frac{\varphi}{c}, \quad \beta=\text { constant }
$$

where the ' + ' sign stands for a right running wave and the '-' sign for a left running wave. Alternatively, we may integrate with the pressure $p$ as independent variable. This gives the following set of differential equations:

$$
\frac{\mathrm{d} u}{\mathrm{~d} p}= \pm \frac{1}{\rho c}, \quad \frac{\mathrm{d} \rho}{\mathrm{d} p}=\frac{1}{c^{2}}, \quad \frac{\mathrm{d} \alpha}{\mathrm{d} p}=\frac{\varphi}{\rho c^{2}}, \quad \beta=\text { constant } .
$$

Here we choose to integrate the form (53). The integration is done by the standard explicit, four-stage Runge-Kutta scheme. The differential equations to be integrated along the left and right subpaths, using the solutions $\boldsymbol{q}^{\mathrm{L}}$ and $\boldsymbol{q}^{\mathrm{R}}$ as the respective initial solutions, are summarized in Fig. 3, together with the explicit Riemann invariant $\beta$. The middle subpath in Fig. 3 shows the explicitly computed Riemann invariants. The latter two imply

$$
\begin{aligned}
& u^{\mathrm{LM}}=u^{\mathrm{RM}}=u^{\mathrm{M}}, \\
& p^{\mathrm{LM}}=p^{\mathrm{RM}}=p^{\mathrm{M}} .
\end{aligned}
$$

Both $u^{\mathrm{M}}$ and $p^{\mathrm{M}}$ are obtained by Newton-Raphson iteration.

For 2D and 3D computations we simply follow the locally 1D approach (directional splitting). For 3D, this implies the wave paths depicted in Fig. 4. Along the left and right subpaths, the extra Riemann invariants $v$ (2D and 3D) and $w$ (3D only) appear. Along the middle subpath, only the multiplicity of the eigenvalue increases with increasing dimensionality.

\subsubsection{Time integration}

For the time integration, use is made of the explicit, third-order accurate, three-stage TVD Runge-Kutta scheme RK3b from [9].

\subsection{Exchange-term evaluation}

For the evaluation of the righthand side of (48a),

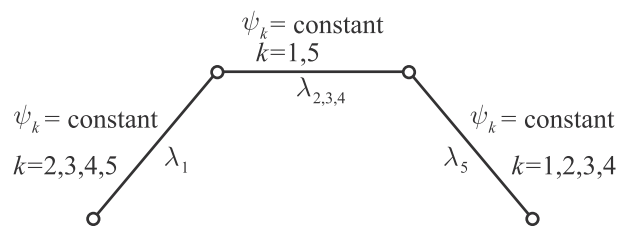

Fig. 2. Wave path in solution space, for 1D physical space, in P-variant ordering, with corresponding Riemann-invariant ordering.

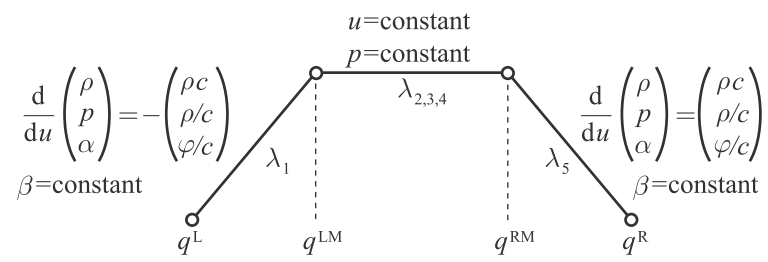

Fig. 3. Wave path in solution space, for 1D physical space, with intermediate solutions $\boldsymbol{q}^{\mathrm{LM}}$ and $\boldsymbol{q}^{\mathrm{RM}}$, ordinary differential equations along left and right subpath, and explicit Riemann invariants along the three subpaths. 


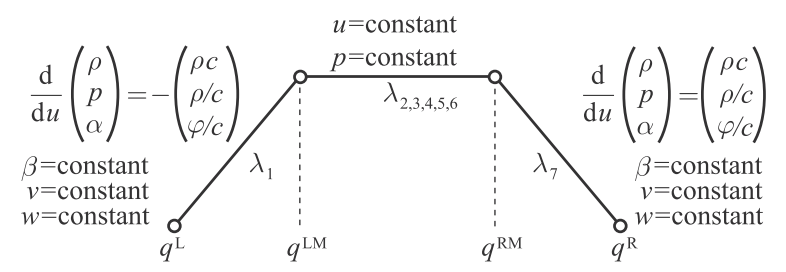

Fig. 4. Wave paths in solution space, for 3D physical space, with intermediate solutions $\boldsymbol{q}^{\mathrm{LM}}$ and $\boldsymbol{q}^{\mathrm{RM}}$, ordinary differential equations along left and right subpaths, and explicit Riemann invariants along the subpaths.

$$
\int_{\Omega} \dot{W} \mathrm{~d} \Omega=\int_{\Omega}(p \boldsymbol{u} \cdot \nabla \alpha+(\alpha-\beta) \boldsymbol{u} \cdot \nabla p+p \varphi \nabla \cdot \boldsymbol{u}) \mathrm{d} \Omega,
$$

we need the spatial distribution of the discrete state variables. For convenience, we start by considering the 1D case. For each of the three terms in the righthand side of (56), two qualitatively different types of contributions may arise when integrating over a finite volume: (i) contributions from the continuous solution in the cell interior and (ii) contributions from the discontinuous solutions at the two cell faces (Fig. 5(a)). The solution at the cell face is denoted by $\boldsymbol{q}^{\mathrm{F}}$. The cell-face contributions can not be ignored. This is obvious when considering the integral over, e.g., the bi-cell $\Omega_{i-1} \cup \Omega_{i}$, in which the integral at cell face $i-\frac{1}{2}$ is included and thus must be evaluated. Since the bi-cell integral can be partitioned as

$$
\int_{\Omega_{i-1} \cup \Omega_{i}} \dot{W} \mathrm{~d} x=\int_{\Omega_{i-1}} \dot{W} \mathrm{~d} x+\int_{\Omega_{i}} \dot{W} \mathrm{~d} x,
$$

this proves that cell-face contributions can not be ignored in single-cell integrals either. Eq. (57) also shows that the contribution to $\int_{\Omega_{i-1} \cup \Omega_{i}}$ of the entire discontinuity at cell face $i-\frac{1}{2}$ has to be split somehow over $\int_{\Omega_{i-1}}$ and $\int_{\Omega_{i}}$. We proceed by presenting a splitting which is consistent with the state distribution in the cell interior and at the cell faces.

At cell faces, the exchange term is not Riemann integrable. We circumvent this difficulty by making consistent use of our Osher-type approximate Riemann solver. I.e., instead of in physical space, at all cell faces, the integration of the exchange term is done in solution space. Per cell we make the following partitioning:

$$
\int_{\Omega_{i}} \dot{W} \mathrm{~d} x=\int_{\boldsymbol{q}_{i-\frac{1}{2}}^{\mathrm{F}}}^{\mathbf{q}_{i-\frac{1}{2}}^{\mathrm{R}}} \dot{W} \mathrm{~d} \boldsymbol{q}+\int_{x_{i-\frac{1}{2}}}^{x_{i-\frac{1}{2}}} \dot{W} \mathrm{~d} x+\int_{\boldsymbol{q}_{i+\frac{1}{2}}^{\mathrm{L}}}^{\boldsymbol{q}_{i-\frac{1}{2}}^{\mathrm{F}}} \dot{W} \mathrm{~d} \boldsymbol{q} .
$$

Only the second integration in the righthand side of (58) is done in physical space, for the continuous solution distribution inside the cell, i.e., the distribution starting with $\boldsymbol{q}_{i-\frac{1}{2}}^{\mathrm{R}}$ at $x_{i-\frac{1}{2}}$ and ending with $\boldsymbol{q}_{i+\frac{1}{2}}^{\mathrm{L}}$ at $x_{i+\frac{1}{2}}$. The first and third integration are

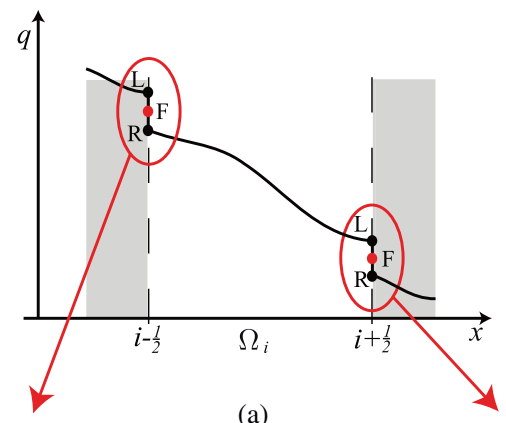

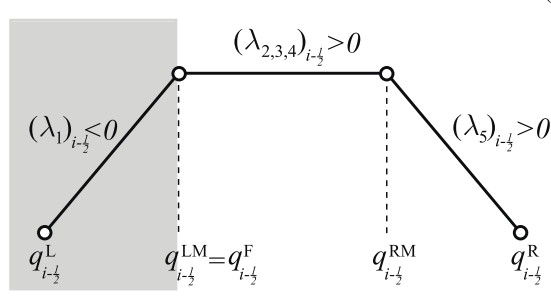

(b)

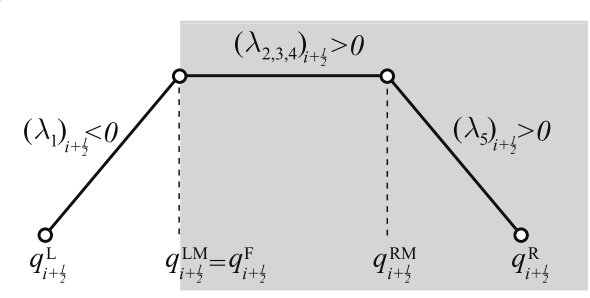

(c)

Fig. 5. Distribution of a solution component in and around finite volume $\Omega_{i}(\mathrm{a})$, together with corresponding wave paths in solution space (in P-variant ordering and for a subsonic flow from the left to the right), at cell faces $i-\frac{1}{2}$ (b) and $i+\frac{1}{2}$ (c). 
done in solution space, along the wave paths, for all waves that propagate from both cell faces into cell $\Omega_{i}$. This is schematically illustrated in Figs. 5(b) and (c), for the example of a subsonic flow in positive $x$-direction.

In both figures, the wave path in solution space has been sketched, with the subpaths in the P-variant ordering. The two subpaths at the right of $\boldsymbol{q}_{i-\frac{1}{2}}^{\mathrm{LM}}=\boldsymbol{q}_{i-\frac{1}{2}}^{\mathrm{F}}$ in Fig. 5(b) correspond with the waves propagating from cell face $i-\frac{1}{2}$ into cell $\Omega_{i}$. The subpath at the left of $\boldsymbol{q}_{i+\frac{1}{2}}^{\mathrm{RM}}=\boldsymbol{q}_{i+\frac{1}{2}}^{\mathrm{F}}$ in Fig. 5(c) corresponds with the wave propagating from cell face $i+\frac{1}{2}$ into cell $\Omega_{i}$. Hence, in evaluating $\int_{\Omega_{i}} \dot{W} \mathrm{~d} x$, at cell face $i-\frac{1}{2}$ the right running waves are taken into account, and at cell face $i+\frac{1}{2}$ the left running waves. This defines the splitting of the exchange-term integral at a single cell face: the part of $\int_{\boldsymbol{q}_{i-\frac{1}{2}}^{\mathrm{L}-\frac{1}{2}}}^{\boldsymbol{q}^{\mathrm{R}}} \dot{W} \mathrm{~d} q$ which corresponds with left running waves (negative eigenvalues) contributes to $\Omega_{i-1}$, and the part corresponding with right running waves (positive eigenvalues) contrib-

utes to $\Omega_{i}$. Contributions due to possible steady waves (zero eigenvalues) may be equally split over both cells.

The state distributions were already obtained in the previous section, so we can proceed by working out the integrals of (58) in more detail.

\subsubsection{Exchange in cell interior}

When using a first-order accurate finite-volume approximation the state values are supposed to be constant in each cell (Fig. 6(a)), implying that the exchange integral over the cell interior equals zero, since all first derivatives of the state variables are zero. In our higher-order accurate spatial discretization the state variables may be supposed to vary piecewise linearly from the cell average in the cell center to the corresponding cell-face values (Fig. 6(b)), causing the spatial derivatives to be nonzero.

In $1 \mathrm{D}$, the integral of the exchange term in the cell interior, the second term in the righthand side of (58), can be written as

$$
\int_{x_{i-\frac{1}{2}}}^{x_{i+\frac{1}{2}}} \dot{W} \mathrm{~d} x=\int_{x_{i-\frac{1}{2}}}^{x_{i}}\left(p u \frac{\partial \alpha}{\partial x}+(\alpha-\beta) u \frac{\partial p}{\partial x}+p \varphi \frac{\partial u}{\partial x}\right) \mathrm{d} x+\int_{x_{i}}^{x_{i+\frac{1}{2}}}\left(p u \frac{\partial \alpha}{\partial x}+(\alpha-\beta) u \frac{\partial p}{\partial x}+p \varphi \frac{\partial u}{\partial x}\right) \mathrm{d} x .
$$

Given the linear distribution of each solution component in each cell half, this integration can be done exactly. As in 1D, where each cell is split in two halves over which the variables are distributed linearly, similarly, in 2D each cell is split in four surfaces over which the variables are distributed linearly, using the cell-center and cell-face-center values as interpolation nodes. Similarly, in 3D the variables are distributed linearly in eight subvolumes of a cell.

\subsubsection{Exchange at cell faces}

As the integration parameter in solution space for the first and third integral in (58), we choose $u$, in consistency with the choice of the independent variable for the system of differential equations (53). Hence, the two aforementioned integrals in (58) can be written as

$$
\begin{aligned}
& \int_{u_{i-\frac{1}{2}}^{\mathrm{F}}}^{u_{i-\frac{1}{2}}^{\mathrm{R}}} \dot{W} \mathrm{~d} u=\int_{u_{i-\frac{1}{2}}^{\mathrm{F}}}^{u_{i-\frac{1}{2}}^{\mathrm{R}}}\left(p u \frac{\mathrm{d} \alpha}{\mathrm{d} u}+(\alpha-\beta) u \frac{\mathrm{d} p}{\mathrm{~d} u}+p \varphi\right) \mathrm{d} u, \\
& \int_{u_{i+\frac{1}{2}}^{\mathrm{L}}}^{u^{\mathrm{F}}}{ }_{\frac{1}{2}} \dot{W} \mathrm{~d} u=\int_{u_{i-\frac{1}{2}}^{\mathrm{F}}}^{u_{i-\frac{1}{2}}^{\mathrm{R}}}\left(p u \frac{\mathrm{d} \alpha}{\mathrm{d} u}+(\alpha-\beta) u \frac{\mathrm{d} p}{\mathrm{~d} u}+p \varphi\right) \mathrm{d} u .
\end{aligned}
$$

Both (60a) and (60b) are evaluated for each of the involved subpaths. Sticking to the example of Fig. 5, subsonic flow from the left to the right at both cell faces $i-\frac{1}{2}$ and $i+\frac{1}{2}$, there is a contribution from the middle subpath at cell face $i-\frac{1}{2}$ only, i.e., to (60a) only. Given the fact that $u$ and $p$ are constant across the middle subpath, the middle-subpath contribution to (60a) reads

$$
\int_{u_{i-\frac{1}{2}}^{\mathrm{LM}}}^{u_{i-\frac{1}{2}}^{\mathrm{RM}}} p u \frac{\mathrm{d} \alpha}{\mathrm{d} u} \mathrm{~d} u=p_{i-\frac{1}{2}}^{\mathrm{M}} u_{i-\frac{1}{2}}^{\mathrm{M}} \int_{\alpha_{i-\frac{1}{2}}^{\mathrm{LM}}}^{\alpha_{i-\frac{1}{2}}^{\mathrm{RM}}} \mathrm{d} \alpha=p_{i-\frac{1}{2}}^{\mathrm{M}} u_{i-\frac{1}{2}}^{\mathrm{M}}\left(\alpha_{i-\frac{1}{2}}^{\mathrm{RM}}-\alpha_{i-\frac{1}{2}}^{\mathrm{LM}}\right)
$$

To evaluate the contributions of the right and left subpaths to (60a) and (60b), respectively, we exploit the ordinary differential equations (53) that determine the corresponding Riemann invariants. This yields

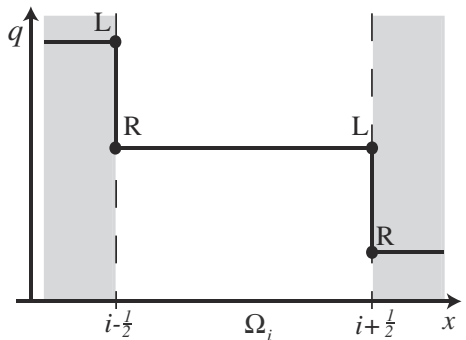

(a)

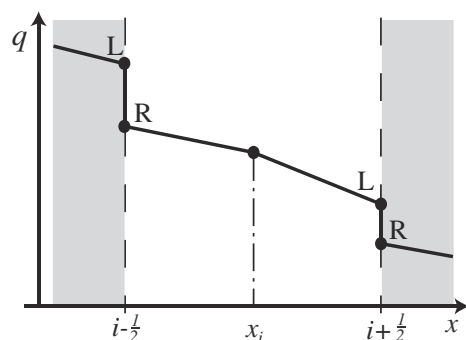

(b)

Fig. 6. First-order accurate (a) and second-order accurate (b) distribution of a solution component in the interior of finite volume $\Omega_{i}$. 


$$
\begin{aligned}
& \int_{u_{i-\frac{1}{2}}^{\mathrm{RM}}}^{u_{i-\frac{1}{2}}^{\mathrm{R}}} \dot{W} \mathrm{~d} u=\int_{u_{i-\frac{1}{2}}^{\mathrm{M}}}^{u_{i-\frac{1}{2}}^{\mathrm{R}}}\left(p \varphi\left(1+\frac{u}{c}\right)+(\alpha-\beta) \rho c u\right) \mathrm{d} u, \\
& \int_{u_{i+\frac{1}{2}}^{\mathrm{L}}}^{u_{i+\frac{1}{2}}^{\mathrm{LM}}} \dot{W} \mathrm{~d} u=\int_{u_{i+\frac{1}{2}}^{\mathrm{L}}}^{u_{i+\frac{1}{2}}^{\mathrm{M}}}\left(p \varphi\left(1-\frac{u}{c}\right)-(\alpha-\beta) \rho c u\right) \mathrm{d} u .
\end{aligned}
$$

Both integrals, (62a) and (62b), are numerically evaluated, using Simpson's 3/8 rule along each of the subpaths. In summary, for the example of subsonic flow from the left to the right, (60a) and (60b) become

$$
\begin{aligned}
& \int_{u_{i-\frac{1}{2}}^{\mathrm{F}}}^{u_{i-\frac{1}{2}}^{\mathrm{R}}} \dot{W} \mathrm{~d} u=p_{i-\frac{1}{2}}^{\mathrm{M}} u_{i-\frac{1}{2}}^{\mathrm{M}}\left(\alpha_{i-\frac{1}{2}}^{\mathrm{RM}}-\alpha_{i-\frac{1}{2}}^{\mathrm{LM}}\right)+\int_{u_{i-\frac{1}{2}}^{\mathrm{M}}}^{u_{i-\frac{1}{2}}^{\mathrm{R}}}\left(p \varphi\left(1+\frac{u}{c}\right)+(\alpha-\beta) \rho c u\right) \mathrm{d} u, \\
& \int_{u_{i+\frac{1}{2}}^{\mathrm{L}}}^{u_{i+\frac{1}{2}}^{\mathrm{F}}} \dot{W} \mathrm{~d} u=\int_{u_{i+\frac{1}{2}}^{\mathrm{L}}}^{u_{i+\frac{1}{2}}^{\mathrm{M}}}\left(p \varphi\left(1-\frac{u}{c}\right)-(\alpha-\beta) \rho c u\right) \mathrm{d} u .
\end{aligned}
$$

In multi-D, the exchange at the cell faces is evaluated in the same way, with as the only difference that the role of the velocity component $u$ is played by the velocity component normal to the cell face.

\section{Numerical results}

In this section we first evaluate the physical model and numerical method presented in the foregoing for three two-fluid shock-tube problems: two interface problems and one mixture problem. Next, more extensively, two standard 2D test cases will be considered: the shock-bubble-interaction problems investigated experimentally by Haas and Sturtevant [7].

Table 1

Initial values for the translating two-fluid interface problem.

\begin{tabular}{llllll}
\hline & $\rho$ & $u$ & $p$ & $\alpha$ & $\beta$ \\
\hline Fluid 1 & 1000 & 1.0 & 1.0 & 1.0 & 1.0 \\
Fluid 2 & 1.0 & 1.0 & 1.0 & 0.0 & 0.0 \\
\hline
\end{tabular}

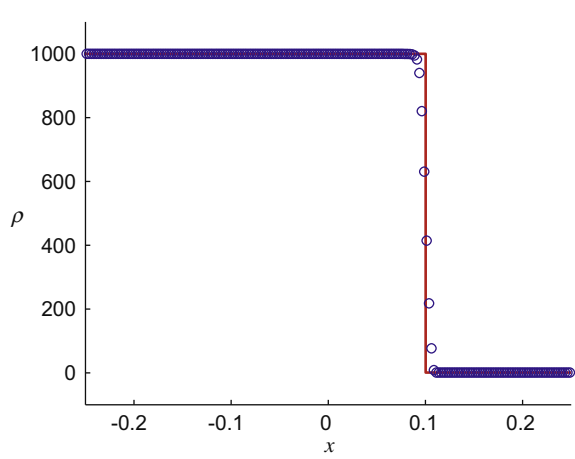

(a) density

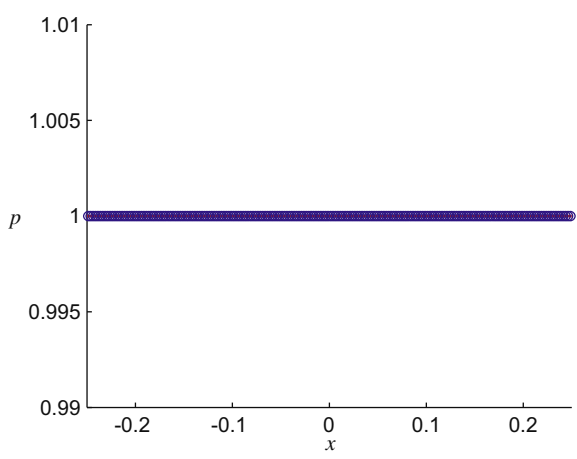

(c) pressure

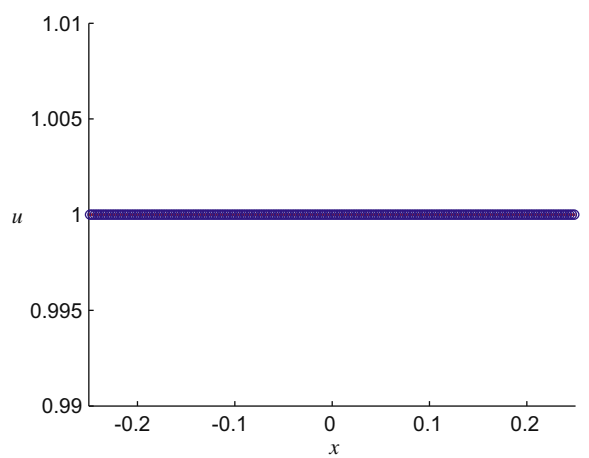

(b) velocity
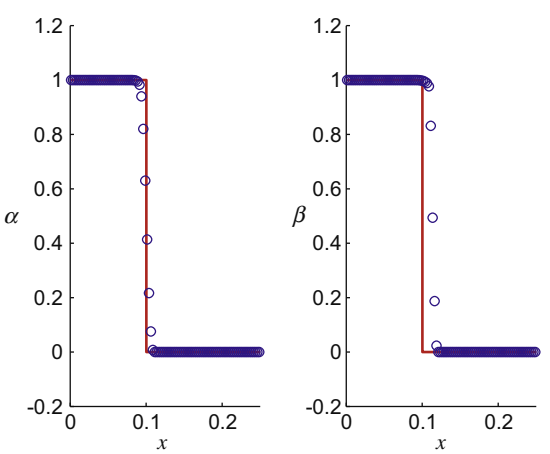

(d) volume fraction and mass fraction

Fig. 7. Numerical " $\bigcirc$ " and exact "__ solution of the translating interface problem at $t=0.1$, for 200 cells and $C F L=0.45$. 
Table 2

Initial values for the no-reflection problem.

\begin{tabular}{llllll}
\hline & $\rho$ & $u$ & $p$ & $\alpha$ & \\
\hline Fluid 1 & 3.1748 & 9.4350 & 100 & 1.0 & 1.0 \\
Fluid 2 & 1.0 & 0.0 & 1.0 & 0.0 & 0.0 \\
\hline
\end{tabular}

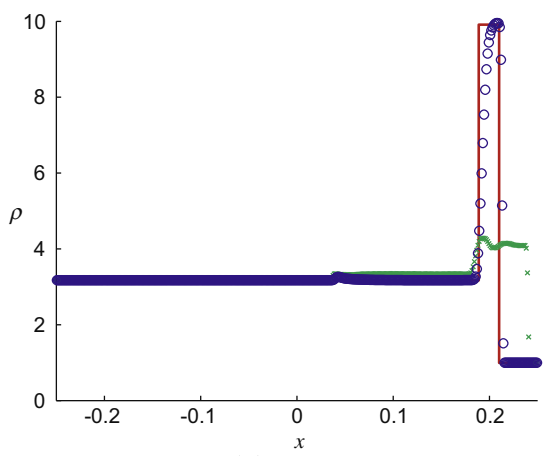

(a) density

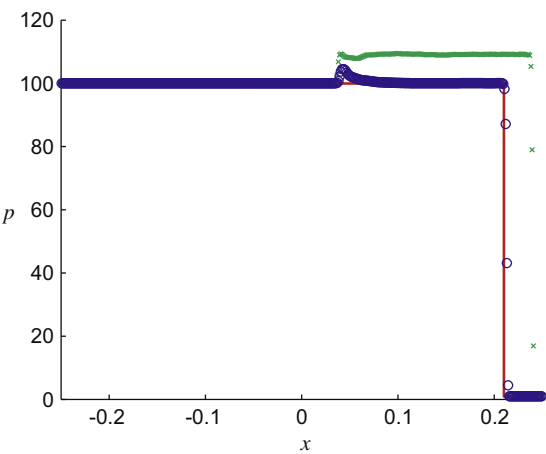

(c) pressure

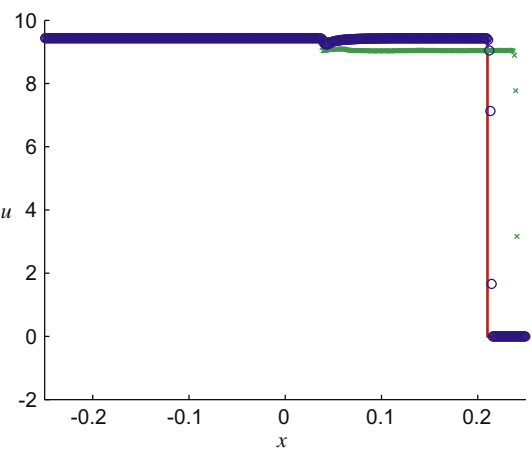

(b) velocity
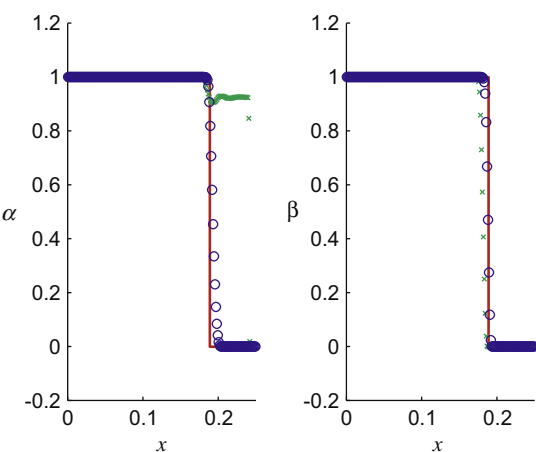

(d) volume fraction and mass fraction

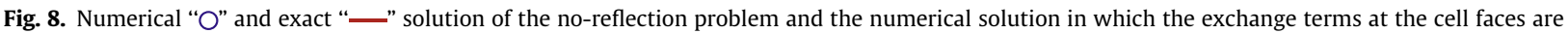
not taken into account " $\times$ ", at $t=0.02$, for 400 cells and $\mathrm{CFL}=0.45$.

\subsection{Shock-tube problems}

\subsubsection{Translating two-fluid interface}

This first test case considers a column of dense perfect gas which pushes a column of 1000 times less dense perfect gas from the left to the right, at uniformly constant speed and pressure. The interest of this test case lies in how well physical model and numerical method can resolve a pure two-fluid interface. The initial conditions are given in Table 1.

Numerical and exact results at $t=0.1$ are given in Fig. 7. The CFL number, given by $\max \left(\frac{\Delta t}{\Delta x}\left|\lambda_{k}\right|_{i+\frac{1}{2}}\right)$, is set equal to 0.45 for all three shock-tube problems. Fig. 7(b) shows that the pressure distribution is perfectly oscillation-free, as it should be. In Fig. 7(d) it seems as if the mass fraction incorrectly resolves the location of the two-fluid interface. This is not the case. In most cells containing both fluids $\beta=\frac{\alpha \rho_{1}}{\rho}=1 /\left(1+\frac{1-\alpha}{\alpha} \frac{\rho_{2}}{\rho_{1}}\right)$ is close to 1 because in most of these cells $\frac{1-\alpha}{\alpha} \frac{\rho_{2}}{\rho_{1}} \ll 1$.

\subsubsection{No-reflection problem}

The second shock-tube case consists of a right state initially at rest and a post-shock state at the left that moves to the right. The test case models the problem of a shock wave running through the left gas column and hitting, at $t=0$, the twofluid interface, which is then still at rest. Again perfect gases are considered.

The initial conditions (Table 2 ) are such that for $t>0$, the two-fluid interface and the transmitted shock wave both move to the right, but that no reflection wave occurs. The latter wave, which also moves to the right because the flow is supersonic, has zero strength.

Correct numerical results (blue) ${ }^{1}$ with corresponding exact results, at $t=0.02$, are given in Fig. 8 . The numerical pressure, density and velocity distributions show a weak reflected wave. Computations at finer grids reveal that it disappears with

\footnotetext{
${ }^{1}$ For interpretation of color in various figures, the reader is referred to the web version of this article.
} 
decreasing mesh width. In Fig. 8 still an erroneous numerical solution is plotted (green). In this solution the exchange-term contributions at the cell faces, as described in Section 3.3.2, were not taken into account. The results clearly show the significance of the cell-face contributions.

\subsubsection{Water-air mixture problem}

The last shock-tube case is a water-air mixture problem, already considered by Murrone and Guillard [14]. This case differs from the previous two in that the complete shock tube is filled with a mixture of the two fluids, i.e., $0<\alpha, \beta<1$. A second difference is that for one of the two fluids, water, the stiffened-gas equation of state is applied,

$$
p=(\gamma-1) \rho e-\gamma \pi
$$

Initially the shock tube is filled with an $\alpha=0.5$ water-air mixture and has a pressure in the left chamber of $10^{4}$ times that of the right chamber. All initial values are given in Table 3, together with the material properties. The dimensions of the quantities $\rho, u, p$ and $\pi$ are $\mathrm{kg} / \mathrm{m}^{3}, \mathrm{~m} / \mathrm{s}$ and $\mathrm{Pa}$, respectively.

The numerical results, at $t=200 \mu$ s are given in Fig. 9. The present numerical results are compared with those from [14]. Both numerical solutions are in perfect agreement. Besides two-fluid interface flows, the current method is also able to simulate flows of two-fluid mixtures.

Table 3

Values for the water-air mixture problem.

\begin{tabular}{|c|c|c|c|c|c|c|c|c|}
\hline \multicolumn{6}{|l|}{ (a) Initial values } & \multicolumn{3}{|c|}{ (b) Material properties } \\
\hline & $\rho$ & $u$ & $p$ & $\alpha$ & $\beta$ & & $\gamma$ & $\pi$ \\
\hline Left chamber & 525 & 0.0 & $10^{9}$ & 0.5 & 0.0476 & Fluid 1 & 1.4 & 0.0 \\
\hline Right chamber & 525 & 0.0 & $10^{5}$ & 0.5 & 0.9524 & Fluid 2 & 4.4 & $6.10^{8}$ \\
\hline
\end{tabular}

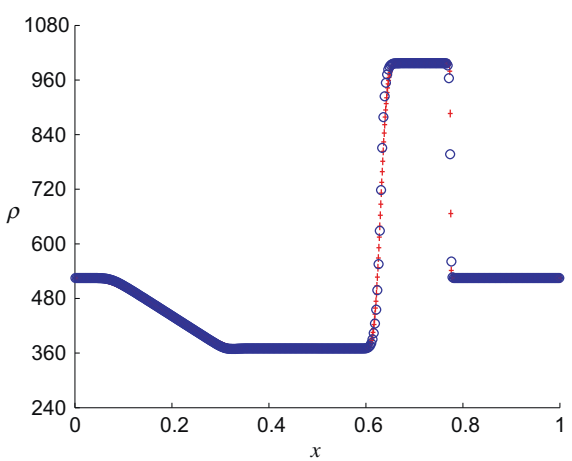

(a) density

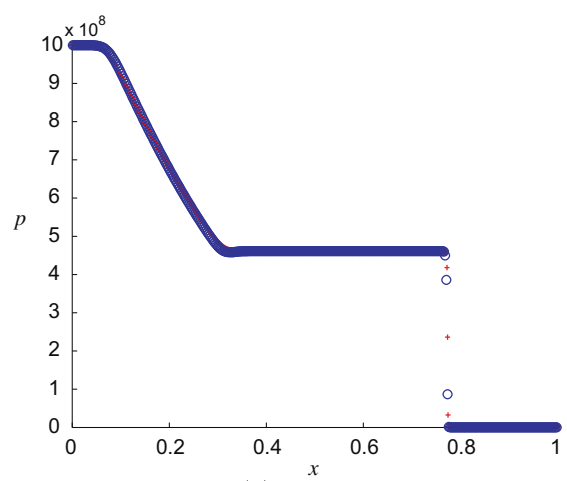

(c) pressure

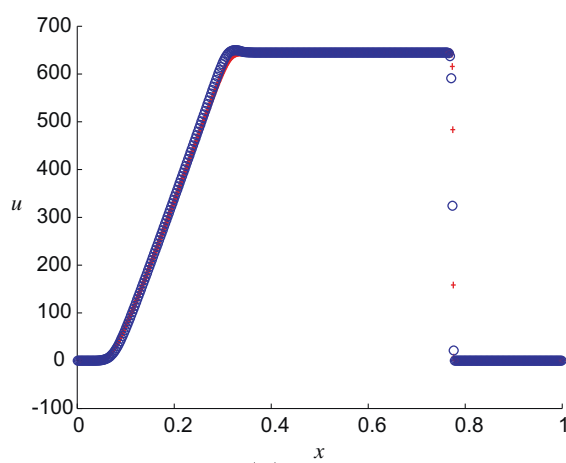

(b) velocity

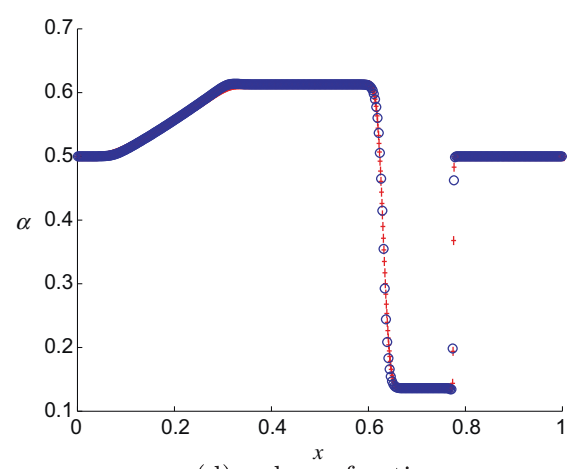

(d) volume fraction

Fig. 9. Present numerical " $O$ " solution for 400 cells and $C F L=0.45$ and numerical solution from [14] " " " of the water-air mixture problem at $t=200 \mu$ s. 


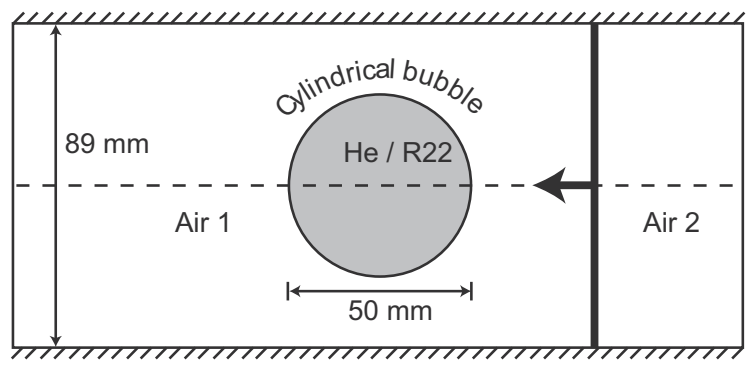

Fig. 10. Initial situation of the shock-bubble-interaction problem, located inside a wind tunnel.

Table 4

Gas properties and initial conditions for the shock-bubble-interaction problems.

\begin{tabular}{lllllll}
\hline & $\gamma$ & $\rho$ & $u$ & $v$ & $p$ & $\beta$ \\
\hline Air 1 & 1.4 & 1.4 & 0.0 & 0.0 & 1.0 & 1.0 \\
Air 2 & 1.4 & 1.92691 & 0.33361 & 0.0 & 1.5698 \\
Helium & 1.648 & 0.25463 & 0.0 & 0.0 & 1.0 \\
R22 & 1.249 & 4.41540 & 0.0 & 0.0 & 0.0 \\
\hline
\end{tabular}

\subsection{Shock-bubble-interaction problems}

The well-documented shock-bubble-interaction experiments performed by Haas and Sturtevant [7] yield excellent benchmarks for evaluating physical models and computational methods for compressible two-fluid flows. Haas and Sturtevant did experiments with spherical and cylindrical bubbles. The latter are considered here.

The cylindrical bubble is filled with a gas which is either lighter or heavier than air, and which is at rest initially. The bubble is surrounded by air which is at rest as well. This state of equilibrium is largely perturbed by a shock wave travelling from the right, through the air, towards the cylindrical bubble (Fig. 10).

The shock hits the bubble. Due to the high velocity of the shock wave and the small size of the bubble (In the experiment, the shock passes the bubble in about $10^{-4} \mathrm{~s}$ ), the two fluids hardly mix. Immiscible, two-fluid flow models are appropriate therefore for these benchmark cases.

The deformation of the cylindrical, two-fluid interface and the resulting wave patterns strongly depend on the kind of gas inside the bubble. Haas and Sturtevant used helium and Refrigerant 22 (R22, a refrigerator gas) for their experiments. The former has a speed of sound higher than that of air, the latter a lower.

We proceed by presenting and discussing our computational results. Comparisons will be made with experimental results. For reasons of computational efficiency, the domain is halved by making use of the symmetry in the flow problems. The domain is divided into $800 \times 200$ rectangular cells. The numerical results in the next two sections are presented in a window containing $420 \times 148$ cells, the same window size as the experimental results. The initial conditions of the different gas states are given in Table 4 . All values of the velocity components are scaled with a speed of sound of $343 \mathrm{~m} / \mathrm{s}$. The helium is slightly contaminated with air, which explains its $\gamma$-value somewhat below $\frac{5}{3}$.

\subsubsection{R22 bubble}

R22 has a higher density and lower ratio of specific heats than air, resulting in an about two times lower speed of sound. The lower speed of sound causes the shock in the bubble, the refracted shock, to lag behind the incoming shock (Fig. 11). Due to the circular shape of the bubble, the refracted shock is curved. For the same reason, the reflected wave, also a shock wave, is curved as well (Fig. 11).

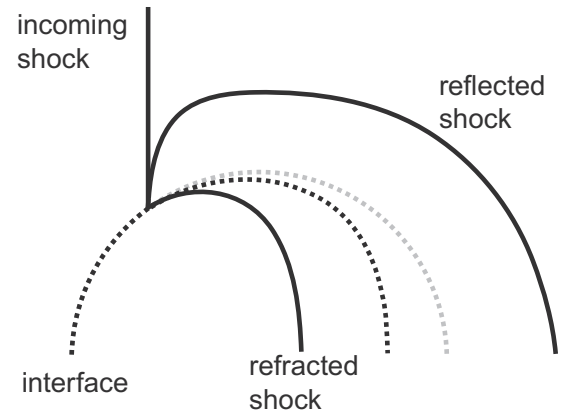

Fig. 11. Wave pattern for the R22 bubble. 



(b)
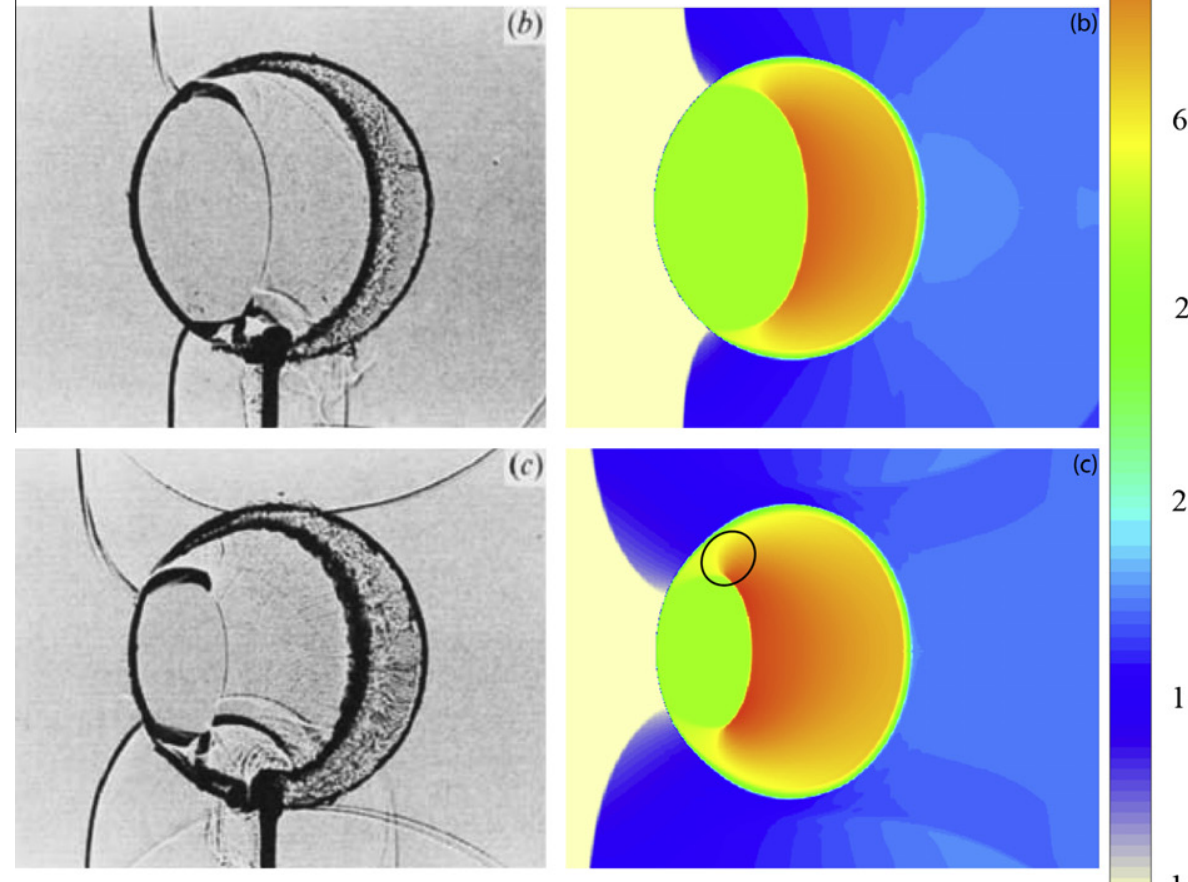

6.1
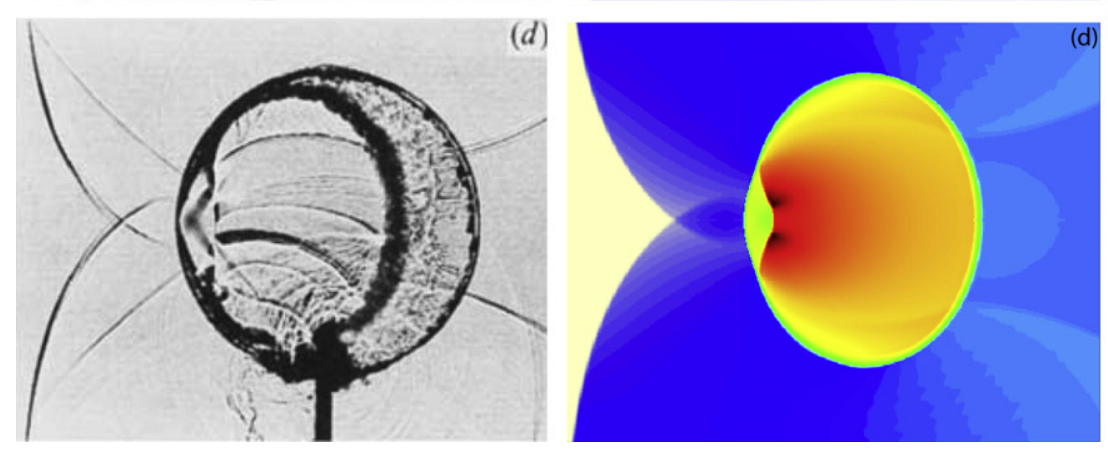

Fig. 12. Evolution of density distribution, R22 bubble, left: shadow-photographs of Haas and Sturtevant [7], right: current numerical results, $t=55 \mu \mathrm{s}$ (a), $115 \mu \mathrm{s}$ (b), $135 \mu \mathrm{s}$ (c), $187 \mu \mathrm{s}$ (d), $247 \mu \mathrm{s}$ (e), $318 \mu \mathrm{s}$ (f) and $342 \mu \mathrm{s}$ (g).

In Fig. 12, we give experimental results obtained by Haas and Sturtevant (the shadow-photographs) and our corresponding numerical results (the color-graphs), showing density distributions. The color-graphs of Figs. 12(b) and (c) show that the incoming and refracted shock are still connected, just as in the corresponding shadow-graphs. The incoming shock becomes curved as well. The refracted shock becomes so much curved that pressure waves arise at the locations of strong curvature (inside black contour line added to color-graph of Fig. 12(c)). Density and pressure strongly increase there (dark spots in color-graph of Fig. 12(d)). The refracted shock finally focuses, resulting in a density peak of more than three times the initial density inside the bubble and a pressure peak of four and a half times the initial pressure. Comparing the color-graphs of Figs. 12(a) and (d), we clearly see that the bubble has been compressed and deformed. Also notice the modest numerical spreading of the two-fluid interface. The agreement between experimental and computational results (shadow-graphs 

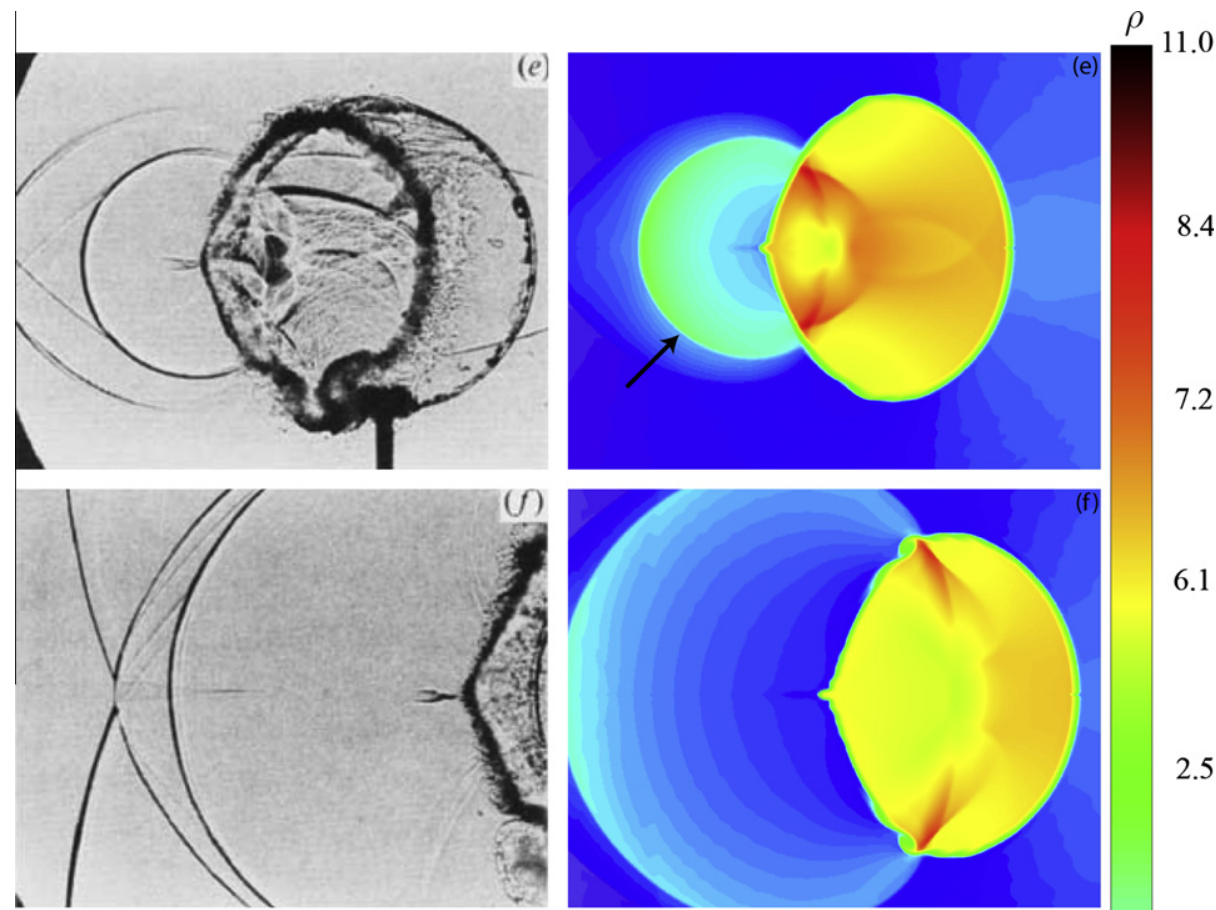

8.4
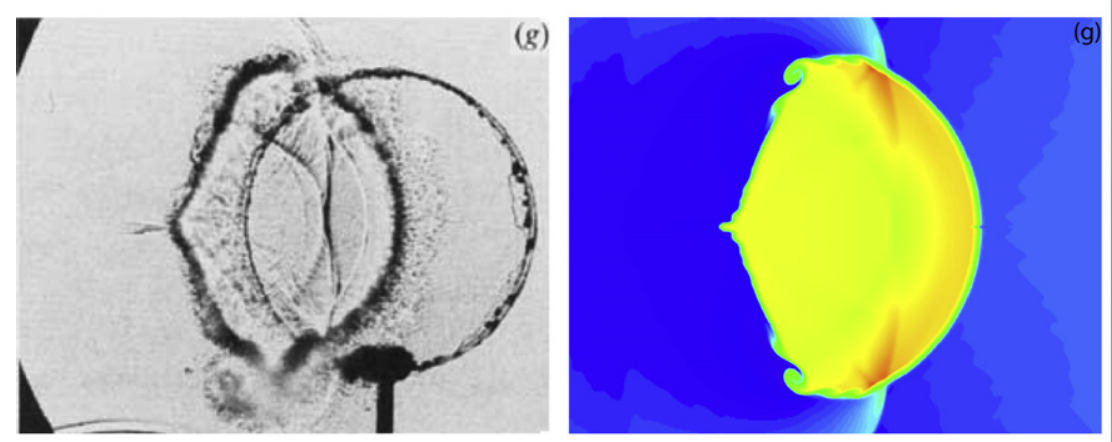

Fig. 12 (continued)

and color-graphs, respectively) is very good. Note that the rather perfect circle which remains to be visible in the shadowgraph is the remainder, on the side wall of the wind tunnel, of the initial bubble's interface (a $0.5 \mu \mathrm{m}$ thick nitrocellulose membrane). At the left of the bubble, the two parts of the incoming shock, that were split at the right of the bubble, meet and cross without affecting each other. On their subsequent paths, they remain attached to the air-R22 interface. After having focused in the most left point of the bubble, the refracted shock expands radially as a transmitted shock (arrow in colorgraph Fig. 12(e)). A high velocity created by the transmitted shock at its central point of origin, causes the interface to bulge out along the symmetry axis (Figs. 12(e)-(g), in both the experimental and the computational results). As the transmitted shock travels along the interface, inward curling vortices are generated at the interface (color-graph in Fig. 12(f), and very clearly in color-graph in Fig. 12(g)); a Richtmyer-Meshkov instability, vaguely visible in the experimental results.

Fig. 13 shows the evolution of the computed pressure and volume-fraction distributions (left and right, respectively) at four instances of time. In the pressure distributions, the location of the two-fluid interface is invisible, as it should be. In the volume-fraction distributions, the white line indicates the initial location of the bubble. Initially, the interface is a single cell thick. When the incoming shock intersects the interface, the latter is smeared over a few cells. At the time of volume-fraction distribution in Fig. 13(b), the refracted shock has almost crossed the bubble. Note that the most left part of the bubble is still in its original position then. Also note the significant compression of the bubble. Going from the volume-fraction distribution in Fig. 13(b) to that in Fig. 13(d), the bubble slightly expands. Fig. 13(c) shows a locally thicker interface at the top (and bottom) of the bubble. This is caused by the incipient rolling-up of the interface. In Fig. 13(d) the interface vortices are clearly visible, and - likewise - the interface bulge along the symmetry axis.

Besides qualitative comparisons between experiment and computation, also quantitative comparisons can be made. In Table 5, following [7], we give: (i) the speed of the incoming shock along the upper boundary of the computational domain, $c_{\mathrm{is}}$, (ii) the speed of the refracted shock, $c_{\mathrm{rs}}$, and (iii) the speed of the right side of the two-fluid interface, $c_{\mathrm{ui}}$, the latter two 


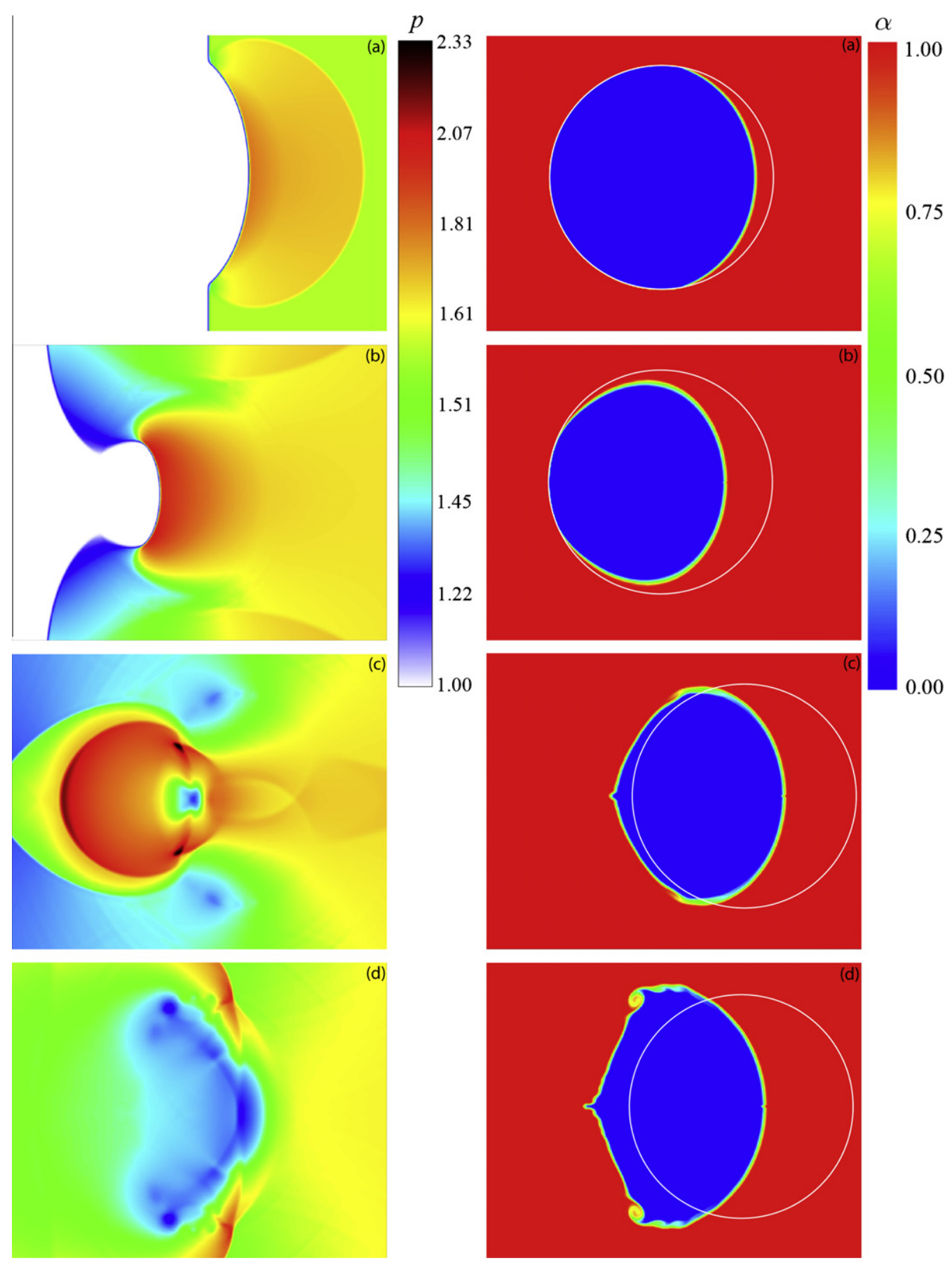

Fig. 13. Computed pressure (left) and volume-fraction (right) distributions, R22 bubble, $t=55 \mu$ s (a), $135 \mu$ s (b), $247 \mu \mathrm{s}$ (c) and $342 \mu \mathrm{s}$ (d). The white circle in the volume-fraction distributions represents the initial bubble.

Table 5

Speeds, in $\mathrm{m} / \mathrm{s}$, of incoming shock, $c_{\mathrm{is}}$, refracted shock, $c_{\mathrm{rs}}$ and right side of interface, $c_{\mathrm{ui}}$, as found in R22 bubble experiment [7] and computations.

\begin{tabular}{llll}
\hline & $c_{\text {is }}$ & $c_{\text {rs }}$ & \\
\hline Haas and Sturtevant [7] & 415 & 240 & 73 \\
Quirk and Karni [18] & 420 & 254 & 70 \\
Present results & 419 & 241 & 75 \\
\hline
\end{tabular}




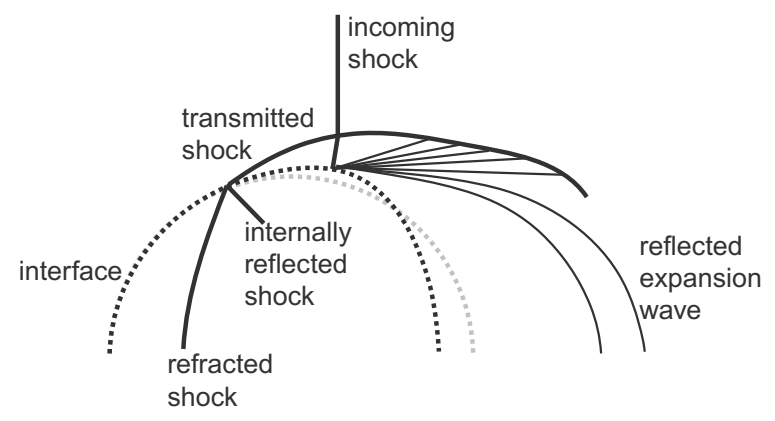

Fig. 14. Wave pattern for the helium bubble.

both along the symmetry axis. Data are given for the three speeds found in the experiment, in the computations of Quirk and Karni [18], and in the present computations. Table 5 shows that the current physical formulation and numerical method perform quantitatively accurate as well.

\subsubsection{Helium bubble}

The second case contains a bubble filled with helium. Helium has a much lower density and a higher ratio of specific heats than air, resulting in a speed of sound higher than air. As a result, the refracted shock runs ahead of the incoming shock (Fig. 14). The reflected wave is an expansion wave.

Depending on the incoming shock wave's strength and angle of incidence onto the interface, the refraction can be regular or irregular. Regular means that incoming wave, refracted wave and reflected wave meet at the same point on the interface. For the R22 bubble the refraction was regular. For the helium bubble the refraction appears to be irregular; the refracted wave intersects the interface ahead of the other waves. In the irregular case, a transmitted shock wave arises where the refracted wave hits the interface. Together with the incoming shock this transmitted shock forms a $\lambda$-shock (Figs. 14 and 15(a)-(c)). Due to the transmitted wave, the part of the incoming shock between the transmitted wave and the interface is deflected. Note the physical correctness of the computational results in this respect, by comparing color-graphs and shadow-graphs in Figs. 15(b) and (c). At the right of the incoming shock the transmitted wave interacts with the reflected expansion wave, causing the transmitted wave to bend inwards (Fig. 14). In the triangular region at the right of the lower part of the incoming wave, and bounded by the transmitted wave and the expansion wave (Fig. 14), the density becomes very high (dark regions in color-graphs of Figs. 15(a)-(c)). Besides the transmitted shock, the refracted shock also causes an internally reflected shock (Fig. 14). The latter is also weakly visible in Figs. 15(b) and (c). In the latter figure, the internally reflected shock has been encircled. The evolution of the internally reflected wave is complex, it has been sketched in Fig. 16. The subnumbers b,c and d in Fig. 16 correspond with those in Fig. 15. The secondary transmitted shock wave, located between first transmitted shock and interface, and sketched in Fig. 16(d), is weakly visible in the color-graph of Fig. 15(d) (jump from yellow to orange). When the first refracted shock leaves the bubble, it continues through the air as a (nearly) circular transmitted wave (Figs. 15(d)-(f)). The compression and deformation of the helium bubble are clearly visible from Figs. 15(a)-(g). In the color-graph of Fig. 15(e), the internally reflected waves have crossed each other and appear as a (light-blue) loop which expands towards the bubble interface. In Fig. 15(f), now also visible in the shadow-graph, the internally reflected wave has completely crossed the interface and continues through the air as a shock wave, which completely encloses the bubble. The bubble starts to roll up (Fig. 15(g)). The rolling-up is caused by a jet along the symmetry axis at the right side of the bubble. From Fig. 15(c) on, when the refracted shock reaches the most left bubble point, the bubble starts to move to the left. This movement is best visible from Figs. 15(f) and (g). For this purpose, the remainders of the initial bubble, on top of the T-shaped support structure in the shadow-graphs, can be taken as a reference.

For the helium-bubble case, pressure and volume-fraction distributions at the same instances of time are given in Fig. 17, pressure in the left graphs, volume fraction in the right graphs. In the aforementioned triangular structure at the right of the incoming shock's lower part, the pressure is much higher than in the surrounding air and in the helium bubble. The evolution of the helium bubble's shape is perfectly shown by the volume-fraction plots. The white line indicates again the initial bubble.

Finally, also for the helium-bubble case some quantitative comparisons can be made of wave speeds. The same speeds are considered as for the R22 bubble. Our computed speeds show a good similarity with the other speeds, especially with those of Quirk and Karni (Table 6).

\section{Conclusions}

In this paper, a new formulation has been derived for Kapila's known five-equation model [10] for compressible, two-fluid Euler flow: a five-equation model that assumes pressure and velocity equilibrium across the two-fluid interface. The current 

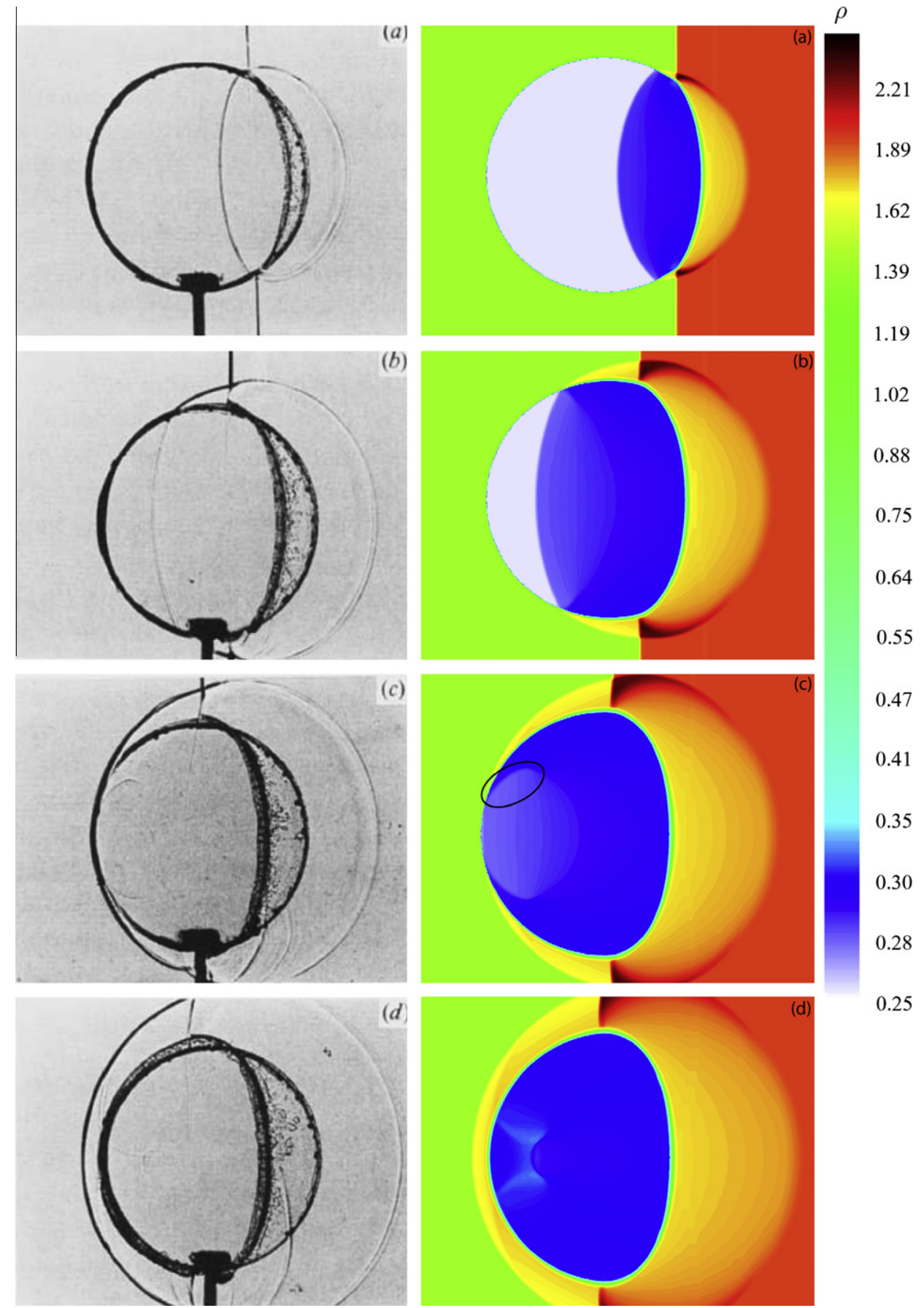

Fig. 15. Evolution of density distribution, helium bubble, left: shadow-photographs of Haas and Sturtevant [7], right: current numerical results, at $t=32 \mu \mathrm{s}$ (a), $52 \mu \mathrm{s}$ (b), $62 \mu \mathrm{s}$ (c), $72 \mu \mathrm{s}$ (d), $82 \mu \mathrm{s}$ (e), $102 \mu \mathrm{s}$ (f) and $245 \mu \mathrm{s}$ (experiment)/ $230 \mu \mathrm{s}$ (computation) (g).

two-fluid flow formulation does not explicitly consider the two-fluid interface. It assumes that the flow is a mixture of the two fluids. The formulation differs from Kapila's in that its fifth equation is a conservation-law-like energy-exchange equation for one of the two fluids. No equation is used to describe the topology of two-fluid interfaces. The complete system of equations is written in integral form, which directly allows for the application of a finite-volume method and Riemann solver.

For the energy-exchange law, two energy-exchange terms have been derived in the paper: a mechanical work term and a thermodynamic work term. The derivation of both terms has been done for smooth flow, and physical interpretations have been made of both terms. Formally, jump conditions also need to be derived for both terms. This has already been done by 


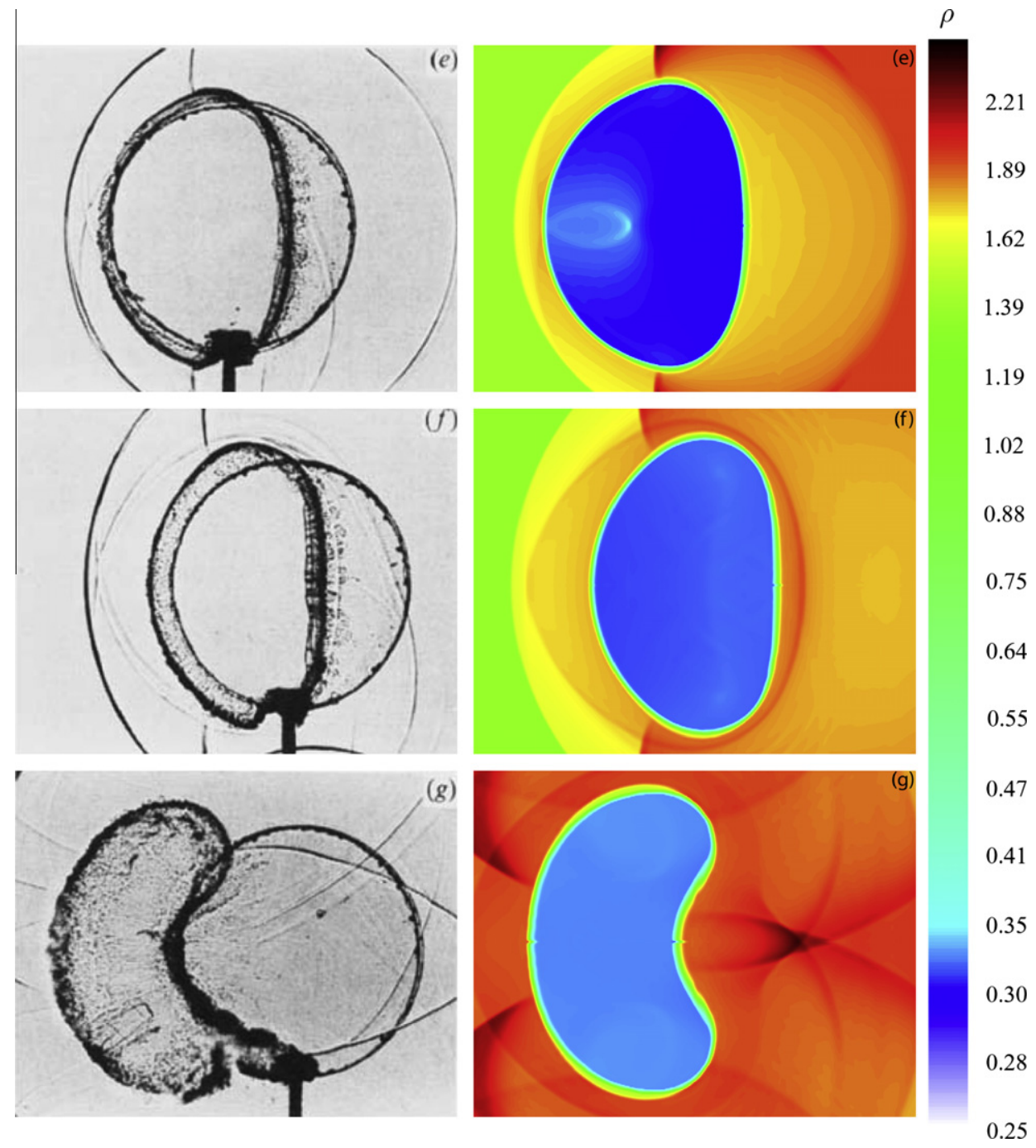

Fig. 15 (continued)

(b)

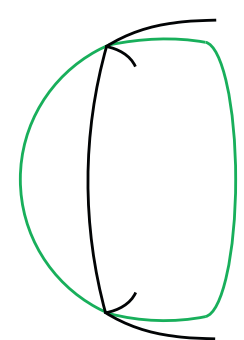

(c)

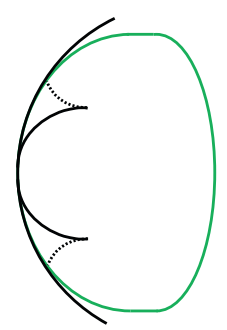

(d)

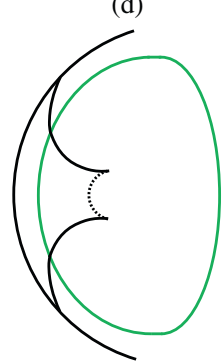

Fig. 16. Schematic drawing of the waves inside the helium bubble, to clarify the color-graphs in Figs. 15b-d. The lines in the plot represent the two-fluid interface "__, relatively strong pressure waves “-” and relatively weak pressure waves “...".

Wackers [28]. An advantage though of our numerical method devised for the reconstruction of the cell-face states and the evaluation of the exchange terms is that it does not need jump conditions.

To evaluate the cell-face states for the fluxes of mass and energy (both of the bulk fluid and of one of the two separate fluids), and the flux of momentum (of the bulk fluid only) and for the evaluation of the energy-exchange terms, an approximate Riemann solver of Osher-type has been constructed. Already for two perfect gases, no explicit expressions can be 


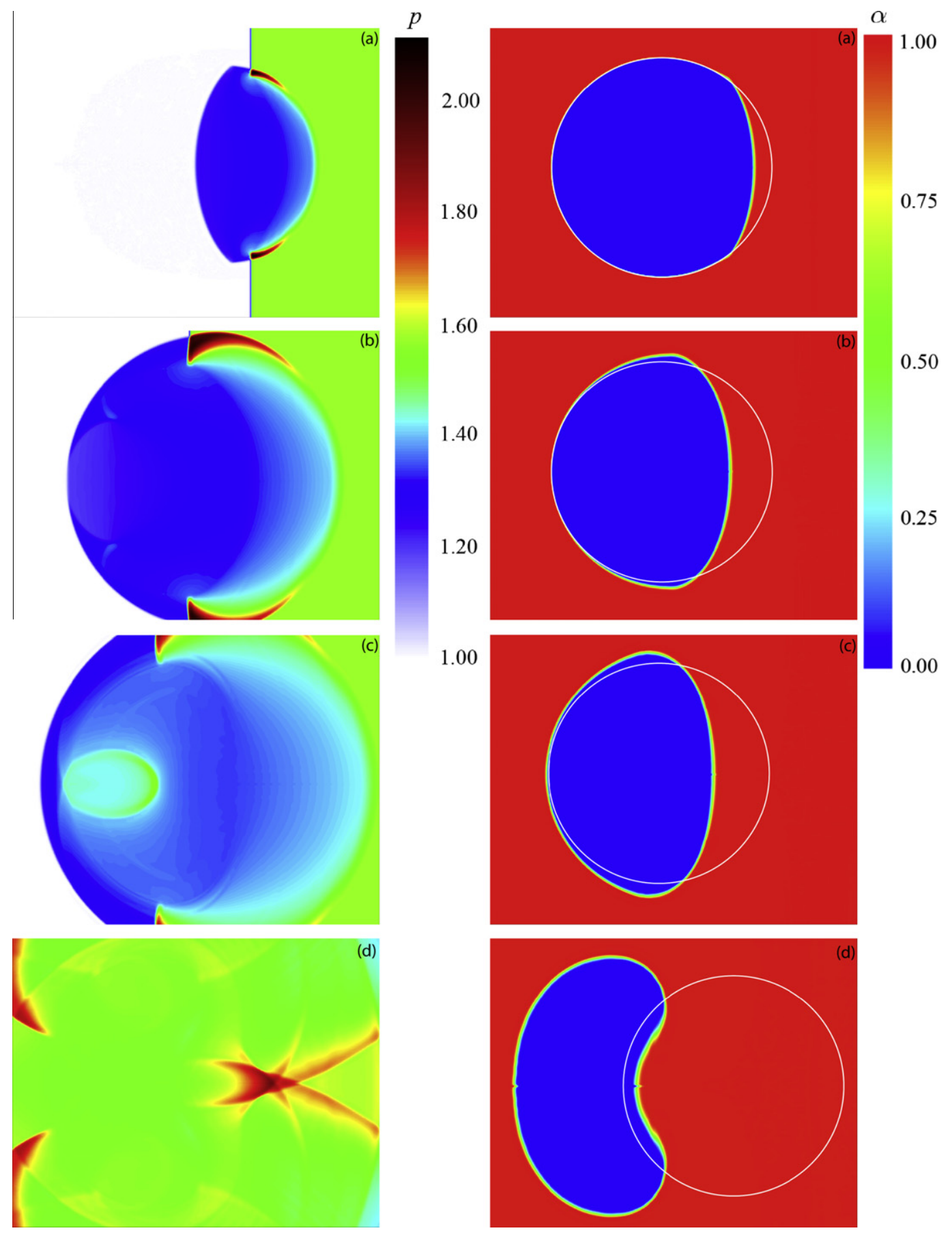

Fig. 17. Computed pressure (left) and volume-fraction (right) distributions, helium bubble, $t=32 \mu \mathrm{s}$ (a), $62 \mu \mathrm{s}$ (b), $82 \mu \mathrm{s}$ (c) and $230 \mu \mathrm{s}$ (d). The white circle in the volume-fraction distributions represents the initial bubble.

Table 6

Speeds, in $\mathrm{m} / \mathrm{s}$, of incoming shock, $c_{\mathrm{is}}$, refracted shock, $c_{\mathrm{rs}}$, and right side of interface, $c_{\mathrm{ui}}$, as found in helium bubble experiment [7] and computations.

\begin{tabular}{llll}
\hline & $c_{\mathrm{is}}$ & $c_{\mathrm{rs}}$ & $c_{\mathrm{ui}}$ \\
\hline Haas and Sturtevant [7] & 410 & 900 & 170 \\
Quirk and Karni [18] & 422 & 943 & 178 \\
Present results & 419 & 956 & 176
\end{tabular}


found for the Riemann invariants needed in the Riemann solver. The ordinary differential equations that implicitly define the Riemann invariants can be easily numerically integrated though. The approximate Riemann solver presented perfectly suits and serves the new formulation of Kapila's five-equation two-fluid model. Left and right cell-face states for the Riemann solver are constructed by a limited, higher-order accurate interpolation.

The Riemann solver is also used for the evaluation of the energy-exchange terms. In a finite-volume setting, the energyexchange terms, which contain first-order spatial derivatives, need to be integrated over the cells, including the cell faces. At the cell faces, the energy-exchange terms are not Riemann integrable. This difficulty is circumvented by integrating the exchange terms at the cell faces in solution space instead of in physical space. For this, consistent and practical use is made of the approximate Riemann solver.

For the time integration, in consistency with the limited, in 1D third-order accurate space discretization, a third-order accurate TVD time integrator is used; an existing, explicit three-stage Runge-Kutta scheme.

The 1D and 2D numerical results presented, show that the method performs well for both two-fluid interface problems and two-fluid mixture problems. Physically correct solutions are obtained without any tuning or post-processing. The method is robust and accurate. Particularly from the 2D test cases, the shock-bubble-interaction problems, it appears that the physical model and numerical method accurately resolve detailed flow features. The two-fluid interface appears to be resolved in accurate position and even rather sharply, despite the use of a mixture model, a model without any explicit description of the two-fluid flow topology.

\section{References}

[1] R. Abgrall, How to prevent pressure oscillations in multicomponent flow calculations: a quasi conservative approach, Journal of Computational Physics 125 (1996) 150-160.

[2] R. Abgrall, V. Perrier, Asymptotic expansion of a multiscale numerical scheme for compressible multiphase flow, SIAM Journal on Multiscale Modeling and Simulation 5 (2006) 84-115.

[3] G. Allaire, S. Clerc, S. Kokh, A five-equation model for the simulation of interfaces between compressible fluids, Journal of Computational Physics 181 (2002) 577-616

[4] M.R. Baer, J.W. Nunziato, A two-phase mixture theory for the deflagration-to-detonation transition (DDT) in reactive granular materials, International Journal of Multiphase Flow 12 (1986) 861-889.

[5] S. Clerc, Numerical simulation of the homogeneous equilibrium model for two-phase flows, Journal of Computational Physics 161 (2000) $354-375$.

[6] H. Guillard, M. Labois, Numerical modeling of compressible two-phase flows, in: P. Wesseling, E. Oñate, J. Périaux (Eds.), ECCOMAS CFD, 2006, <http:// proceedings.fyper.com/eccomascfd2006/>.

[7] J.F. Haas, B. Sturtevant, Interaction of weak shock waves with cylindrical and spherical gas inhomogeneities, Journal of Fluid Mechanics 181 (1987) 41 76.

[8] P.W. Hemker, S.P. Spekreijse, Multipe grid and Osher's scheme for the efficient solution of the steady Euler equations, Applied Numerical Mathematics 2 (1986) 475-493.

[9] W. Hundsdorfer, B. Koren, M. van Loon, J.G. Verwer, A positive finite-difference advection scheme, Journal of Computational Physics 117 (1995) $35-46$.

[10] A.K. Kapila, R. Menikoff, J.B. Bdzil, S.F. Son, D.S. Stewart, Two-phase modeling of deflagration-to-detonation transition in granular materials: reduced equations, Physics of Fluids 13 (2001) 3002-3024.

[11] B. Koren, A robust upwind discretization method for advection, diffusion and source terms, in: C.B. Vreugdenhil, B. Koren (Eds.), Notes on Numerical Fluid Mechanics, vol. 45, Vieweg, 1993, pp. 117-138.

[12] J. Massoni, R. Saurel, B. Nkonga, R. Abgrall, Propositions de méthodes et modèles Eulériens pour les problèmes à interfaces entre fluides compressibles en présence de transfert de chaleur, International Journal of Heat and Mass Transfer 45 (2002) 1287-1307.

[13] W.A. Mulder, S. Osher, J.A. Sethian, Computing interface motion in compressible gas dynamics, Journal of Computational Physics 100 (1992) $209-228$.

[14] A. Murrone, H. Guillard, A five equation reduced model for compressible two phase flow problems, Journal of Computational Physics 202 (2005) $664-$ 698.

[15] S. Osher, R.P. Fedkiw, Level Set Methods and Dynamic Implicit Surfaces, Springer, 2003.

[16] S. Osher, F. Solomon, Upwind difference schemes for hyperbolic systems of conservation laws, Mathematics of Computation 38 (1982) 339-374.

[17] F. Petitpas, E. Franquet, R. Saurel, O. Le Metayer, A relaxation-projection method for compressible flows. Part II: Artificial heat exchanges for multiphase shocks, Journal of Computational Physics 225 (2007) 2214-2248.

[18] J.J. Quirk, S. Karni, On the dynamics of a shock-bubble interaction, Journal of Fluid Mechanics 318 (1996) 129-163.

[19] E. Romenski, A.D. Resnyansky, E.F. Toro, Conservative hyperbolic formulation for compressible two-phase flow with different phase pressures and temperatures, Quarterly of Applied Mathematics 65 (2007) 259-279.

[20] R. Saurel, R. Abgrall, A multiphase Godunov method for compressible multifluid and multiphase flows, Journal of Computational Physics 150 (1999) $425-467$.

[21] R. Saurel, E. Franquet, E. Daniel, O. Le Metayer, A relaxation-projection method for compressible flows. Part I: The numerical equation of state for the Euler equations, Journal of Computational Physics 223 (2007) 822-845.

[22] R. Saurel, F. Petitpas, R.A. Berry, Simple and efficient relaxation methods for interfaces separating compressible fluids, cavitating flows and shocks in multiphase mixtures, Journal of Computational Physics 228 (2009) 1678-1712.

[23] J.A. Sethian, Level Set Methods and Fast Marching Methods: Evolving Interfaces in Computational Geometry, Fluid Mechanics, Computer Vision and Materials Science, Cambridge University Press, 1999.

[24] H.B. Stewart, B. Wendroff, Two-phase flow: models and methods, Journal of Computational Physics 56 (1984) 363-409.

[25] P.K. Sweby, High resolution schemes using flux limiters for hyperbolic conservation laws, SIAM Journal on Numerical Analysis 21 (1984) 995-1011.

[26] E.H. van Brummelen, B. Koren, A pressure-invariant conservative Godunov-type method for barotropic two-fluid flows, Journal of Computational Physics 185 (2003) 289-308.

[27] B. van Leer, Upwind-difference methods for aerodynamic problems governed by the Euler equations, in: B.E. Engquist, S. Osher, R.C.J. Somerville (Eds.), Lectures in Applied Mathematics, vol. 22, American Mathematical Society, 1985, pp. 327-336.

[28] J. Wackers, Surface Capturing and Multigrid for Steady Free-Surface Water Waves, Ph.D. Thesis, Delft University of Technology, 2007, <http:// repository.tudelft.nl/>.

[29] N.P. Waterson, H. Deconinck, Design principles for bounded higher-order convection schemes - a unified approach, Journal of Computational Physics 224 (2007) 182-207. 